\title{
On the use of small ring testing for the characterisation of elastic and yield material property variation in additively manufactured materials
}

\author{
J. P. Rouse ${ }^{* 1}$, M. Simonelli ${ }^{2}$ and C. J. Hyde ${ }^{1}$ \\ ${ }^{1}$ Gas Turbine and Transmission Research Centre (G2TRC), University of Nottingham, \\ Nottingham, NG7 2TU, UK \\ ${ }^{2}$ Centre for Additive Manufacturing, University of Nottingham, Nottingham, NG7 2TU, UK
}

\begin{abstract}
The present work introduces a small volume, high throughput testing method that may be used to quickly, and with high spatial fidelity, investigate local material properties in additively manufactured materials. A case study application is presented here, considering components made from the alloy Ti-6Al-4V by the powder bed fusion process. Novel tensile small ring samples are produced at multiple locations over the print volume and are tested in order to estimate local tensile material responses. An inverse method is implemented in order to estimate representative material properties at each testing location by optimising initial values against sequential finite element model results. Initial estimates themselves are found through a geometrically non-linear semi-analytical model that approximates elastic material response. A good level of repeatability is noted between small ring tests conducted at specific build locations, suggesting a limited effect of build height on constitutive response and robust testing/analysis methodology. Small ring tests indicate a mean Young's modulus of $82.83 \mathrm{GPa}$ (with a standard deviation of $4.57 \mathrm{GPa}$ ) and a mean yield stress of $655.58 \mathrm{MPa}$ (with a standard deviation of $115.73 \mathrm{MPa}$ ). Full sized "conventional" tests, published in the author's previous work, indicate a Young's modulus of $114.45 \mathrm{GPa}$ and a yield stress of 771.266 MPa. Limited fractography has indicated that there is a wide variation in porosity across the build volume, suggesting that the deviations in local material properties are due to the use of a reduced print volume in specimen manufacture.
\end{abstract}

Keywords: Ti-6Al-4V, Additive Manufacture, Small Ring Testing, Reduced Build Volume, Mechanical Characterisation, Laser Powder Bed Fusion.

\section{Nomenclature}

$\begin{array}{ll}t & \text { Small ring (axial) thickness }(2 \mathrm{~mm}) \\ w & \text { Small ring (radial) width }(1 \mathrm{~mm}) \\ R_{I} & \text { Small ring internal radius }(4.5 \mathrm{~mm}) \\ P & \text { Pin reaction force }(\mathrm{N}) \\ \Delta_{0} & \text { Initial pin separation }(\mathrm{mm}) \\ \Delta & \text { Pin separation }(\mathrm{mm}) \\ Q & \text { Dummy horizontal pin reaction force }(\mathrm{N}) \\ M_{0} & \text { Reaction bending moment at pin }(\mathrm{Nm}) \\ a & \text { Instantaneous major elliptical ring radius }(\mathrm{mm})\end{array}$

*Corresponding Author - James Rouse, Gas Turbine and Transmission Research Centre (G2TRC), University of Nottingham, Nottingham, NG7 2TU, UK Email: James . Rouse@nottingham. ac . uk Tel: +44 (0)115 8467683 


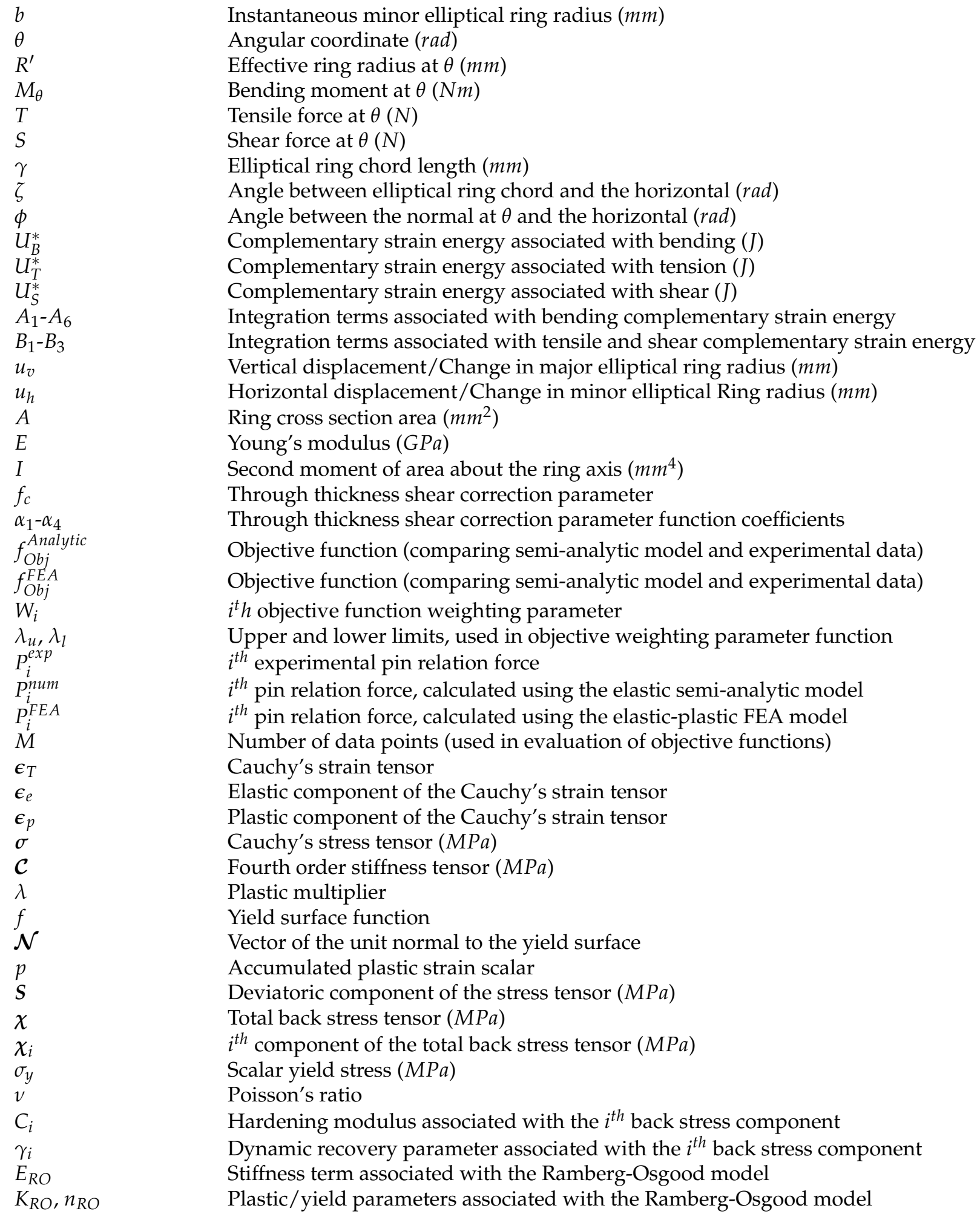

\section{Introduction}

It is well known that additive manufacture has the potential to revolutionise how engineers design and produce components in systems that are vital for modern life. New areas of the design space will become available when the restrictions of subtractive manufacturing are removed, allowing optimum 
component geometries to be achieved and material waste to be minimised [1,2]. The societal impacts of enabling titanium alloy additive manufacture will be particularly amplified due to the applications where such materials are typically deployed, notably aerospace and biomedical. In these safety critical situations it is vital that material properties over build volumes are known, consistent, and confidently repeatable. Laser powder bed fusion (LPBF) is a key process in the field of additive manufacturing which can, in principle, be exploited to produce parts with any internal or external geometry, thereby broadening the range of possible design solutions [2, 3, 4]. Many adaptations have been made in order to improve the quality of prints and reduce process times since the inception of LPBF. Examples of these developments include the introduction of multiple-laser machines which reduced processing time and offer a greater degree of freedom in terms of in process heat treatment, reduced bed volumes (RBV) platforms which limit powder waste, and techniques which result in improved powder layer uniformity. Additive processes, such as LPBF, also have the advantage of circumventing difficulties faced in the forming of high strength materials, such as Nickel-based superalloys, by conventional means. By way of example, the poor machinability of such materials, which can affect the surface quality of manufactured parts and lead to under-performance of components, can in part be mitigated through additive process.

LPBF is not without its challenges. It has been well documented, for example, that parts manufactured via LPBF exhibit a reduction in apparent mechanical strength and stiffness when compared with equivalents machined from conventional (e.g. wrought) materials $[5,6,7,8,9]$. A further concern with the LPBF process is the uncertainty over consistency in the microstructural and mechanical properties produced across the build volume [10]. This potential lack of material consistency is not a concern within conventionally manufactured, e.g. wrought, materials, for which the manufacturing processes are well established. Developing an understanding of how processing parameters affect the mechanical characteristics of AM materials is also of great interest. This is particularly true of Ti-6Al-4V (the focus of the present work) due to its poor thermal conductivity and chemical reactivity to oxygen [11]. Indeed, the effects of processing history on $\mathrm{AM} \mathrm{Ti}-6 \mathrm{Al}-4 \mathrm{~V}$ has recently received a great deal of attention in the literature. Liu and Shin, for example, compared microstructures and mechanical properties for Ti-6Al-4V in directed energy deposition, laser powder bed fusion (often erroneously refereed to as selective laser melting, or SLM), and an electron beam melting processes [11]. It was noted that, due to differences in cooling rates and process temperatures, LPBF leads to acicular $\alpha^{\prime}$ martensitic structure, whereas EBM leads to $\alpha+\beta$ lamellar structure free from residual stresses. The presence of a Martensitic structures tends to increase yield and ultimate tensile strength but reduce material ductility. Recently, Ettefagh at al. studied influence of heat treatment on the corrosion behaviour of LPBF Ti-6Al-4V [12]. In this work, corrosion rates were almost sixteen times greater for as manufactured samples than in cold rolled samples, although it was demonstrated that application of the correct heat treatment could reduce the disparity. Mechanical properties of LPBF manufactured Ti-6Al-4V were very recently evaluated by Formanoir and co-workers [13]. So called "hull-bulk" build strategies were considered, wherein a different layer thickness was used in the bulk (centre) of a part, compared to the hull (outer layer). It is important to note LPBF Ti-6Al-4V is used as a demonstrative example in the present work; the small ring testing method is applicable to many additive manufacturing testing challenges, such as those considered by Formanoir et al. The many challenges and considerations relating to LPBF summarised above will likely be addressed in a variety of ways, such as powder developments, process parameter optimisations, and optimised microstructures. By whatever means the integrity of LPBF materials is improved however, large amounts of mechanical testing will be required in order to gain confidence over a particular remedy's efficacy.

A methodology used for mechanical testing of LPBF materials will need to satisfy several criterion; it will need offer high throughput (enabled through both specimen design and test configuration) so that print process variants can be evaluated while simultaneously utilising small enough test samples such that local properties in the build volume can be explored and consistency verified. The present work develops such a testing methodology by utilising a novel "small specimen" geometry, namely the small ring. The testing method is boasts a large equivalent gauge length (thereby reducing the errors in strain state caused by displacement measurement uncertainty) and a simple and fast specimen preparation and test procedure that can be easily implemented on almost any uniaxial testing frame. An inverse finite element method is used here to estimate tensile mechanical properties based on ring sample responses taken across a build volume, thereby establishing an interpretation and constitutive relations approximation method for tensile small ring results. The inverse method in turn uses a new semi-analytical geometrically non-linear model to estimate elastic materials properties. 
Small specimen test techniques have been developed over the past 25-30 years in order to address the challenge of assessing material properties/condition in situations when there is not enough material to manufacture standard, full-size, test specimens. A small specimen may be roughly defined as one having a major dimension no greater than $20 \mathrm{~mm}$ (note conventional "dog bone" testing samples typically have major dimensions over $100 \mathrm{~mm}$ ). Such applications include:

- the development new materials from which only small amounts can be achieved [14].

- when investigating the condition of in-service components, such as power plant steam pipes [15], where the removed material volume must not be so large so as to have a significant detrimental effect on the component strength.

- when evaluating service-aged components, such as turbine blade roots [16], coatings [17] or heat-affected-zones (HAZ) in welds [18], where the material zones of interest may be limited.

- the evaluation of additively manufactured materials [19, 20,21, 22], as discussed above.

Small specimens can be used to acquire properties related to a range of physical phenomenon such as tensile behaviour [23, 24], fatigue [25], fracture [26], crack propagation [27], and creep [28, 29]. A number of small specimen types have been developed [29], including the small ring specimen, initially devised in 2009 by Hyde and Sun [30] for the evaluation of creep properties and since developed by Kazakeviciute et al. [31] for the evaluation of tensile properties of materials. This small specimen type has several advantages over alternative methods, such as having a large equivalent gauge length (approximately $50 \mathrm{~mm}$, providing high test sensitivity), being self-aligning in nature, demonstrably insensitive friction conditions at the "grips" (pins), and simple to manufacture. An excellent example of the application of small specimens for the evaluation of AM material properties can be found in the work from Sandia National Laboratories [32, 33], wherein "cassettes" containing over 100 tensile ("dog bone") samples are printed and tested individually using a bespoke grip arrangement. Importantly, the arrangement used in Sandia's methodology allowed test programs to be completed in a very short amount of time with almost no manual intervention. The methodology discussed in the present work (utilising "small rings") is an alternative to the Sandia method that, while requiring a far greater degree of manual intervention between tests, exhibits a large equivalent specimen gauge length and hence increased confidence in strain states (i.e. the effect of uncertainty in displacement measurements is reduced). Furthermore, the small ring method discussed here can be readily implemented on almost any uniaxial test machine through the use of a modified conventional uniaxial specimen (see section 2.2). Methods such as those developed by Sandia require bespoke testing frame designs in order to offer the level of testing automation that makes them attractive. Crucially, both methodologies allow for localised evaluation of material properties using simple specimen geometries that can be manufactured and tested quickly. Small rings are favoured over miniature tensile specimens in the present work due to the ease of testing, large equivalent gauge length, and self aligning nature. The more complex stress state induced in small ring specimens does complicate data interpretation, however it has been shown in the authors' previous work [31] that the specimen is sensitive to the material and the the inverse method developed in the present work provides an approach to extract constitutive parameters. The work of Lancaster and co-workers also deserves particular attention here [34, 35], where small punch test based methodologies were developed for characterising tensile and fatigue properties. These methods have been applied in investigations of Ti-6Al-4V behaviours. The small punch methods used in the work of Lancaster and the small ring procedure utilised here share many features; both implement simple geometries at a small scale to determine macroscopic representative properties.

In this study, small ring specimens have been used to determine the tensile properties of Titanium alloy Ti-6Al-4V manufactured by LPBF over an RBV. Of particular interest in the present work are variations in material properties over the build volume (both in the build plane and vertically), leading to the adoption of small ring testing techniques to explore material properties at a much finer spatial resolution than would be possible with conventional specimens. While the focus of the present work is the tensile behaviour of LPBF Ti-6Al-4V, it is worth noting that the small ring testing approach developed here is applicable in many areas. The high throughput and repeatable nature of the testing method will, for example, be of great benefit in ranking powder degradation quantification, assessing print parameter and powder variants, and screening new materials/compositions. 


\section{Experimental Methodology}

\subsection{Specimen Manufacture}

The material used in this research was Ti-6Al-4V grade 23 in the form of pre-alloyed powders of spherical morphology and with size distribution $D 10=22.1 \mu \mathrm{m}, D 50=33.5 \mu \mathrm{m}$ and $D 90=49.9 \mu \mathrm{m}$ (supplied by Carpenter AM).

Twelve tubes were manufactured using the reduced build volume (RBV) configuration of the Renishaw AM400, thereby providing "blanks" which could be sectioned in order to form the small ring samples. For sake of simplicity the specimens were numbered consistently with the laser scanning order (this can be seen in figure 1). A comparison of the printed tube and the small ring sample (sectioned from the tube) can be seen in figure 2 (a). All tubes were printed with an internal radius, $R_{I}$ (see figure 2 (b)), of $4.5 \mathrm{~mm}$, a wall thickness $(W)$ of $1 \mathrm{~mm}$, and a thickness $(t)$ of $2 \mathrm{~mm}$. The tubes were printed in the z-orientation (i.e. with the main axis parallel to the building direction as per ISO / ASTM52921, see figure 2 (b)) on $3 \mathrm{~mm}$ supporting structures that facilitated their detachment from the build plate at the end of the printing process. The AM400 was operated with a laser power of $300 \mathrm{~W}, 70 \mu \mathrm{m}$ hatch spacing, $50 \mu \mathrm{m}$ layer thickness, and $670 \mathrm{~mm} / \mathrm{s}$ scan speed (point distance of $40 \mu \mathrm{m}$ ). Each layer was melted once. The laser scan direction was then rotated by $67 \mathrm{deg}$ between each two consecutive layers to randomise the scan vector length per cross-section. All samples were built with identical process parameters on Ti-6Al-4V build plates which were not preheated. Samples were fabricated across a square build plate (approximately $70 \mathrm{~mm}$ ) to investigate the effect of the specimen position on quality. The process chamber atmosphere was regulated with circulating Argon to keep oxygen levels below $0.09 \%$. Note that, for specimen identification purposes, the vertical locations of the small ring specimens (in the source tubes) were noted and tracked throughout testing (the labelling convention figure 2 (b) was adopted). Orientation marks were also made on the tube and maintained when ring specimens were parted off. These orientation marks were then used during the experimental setup to ensure loading directions were common for all rings, regardless of source location in the print volume. Tubes were turned on a mandrel so that rings could be parted off, with the final thickness dimension controlled by hand polishing.

Prior to any mechanical testing and machining, all the tubular samples were subject to heat treatment. The specimens were enclosed in evacuated quartz tubes and then annealed at $940^{\circ} \mathrm{C}$ for 2 hours followed by an aging treatment at $700^{\circ} \mathrm{C}$ for 4 hours as per ASTM F3001 - 14 . The thermal and processing histories experienced by specimens will, at least in part, be dependent on their respective build height positions (see figure 2 (b)). In the present work, heat treatments have been applied to the AM tubes prior to machine to normalise the microstructure, relieve residual stresses, and hence reduce this build direction variability. It is important to note however that the small ring testing procedure developed here offers a high spatial fidelity, thereby enabling investigations in cross build volume material variations (where they exist). After heat treatment the specimens were air cooled whilst remaining enclosed in the evacuated tubes to limit the potential for oxidisation. 


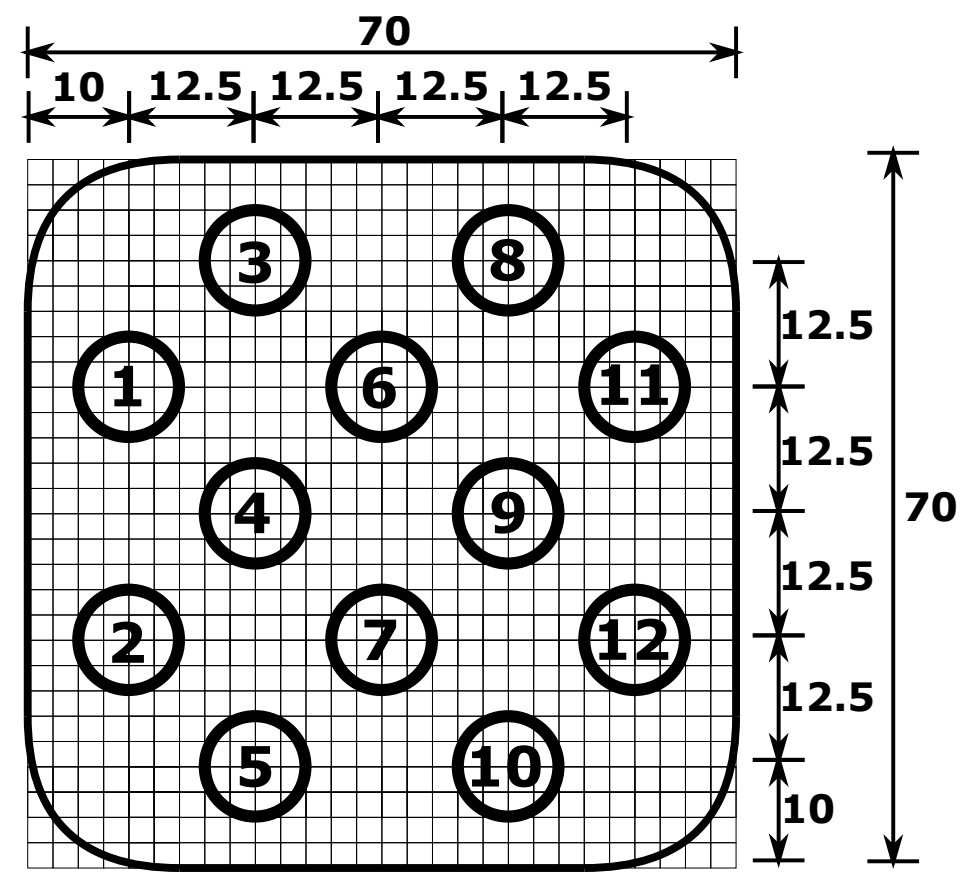

Figure 1: The build positions of the 12 Ti-6Al-4V "tubes" on the reduced build volume (all dimensions are given in $\mathrm{mm}$ ). 


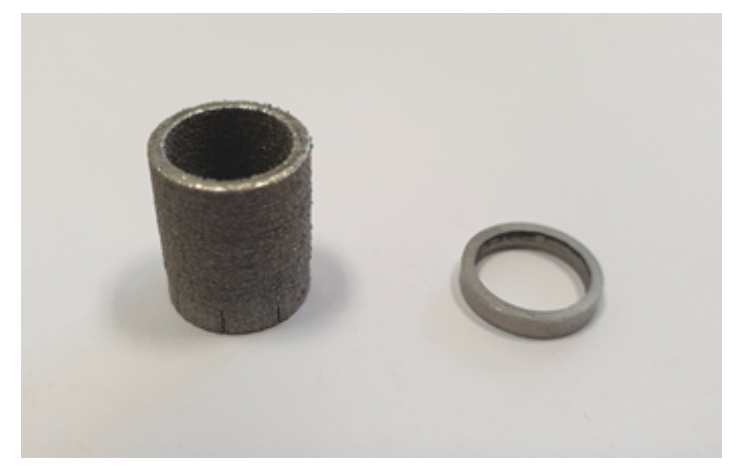

(a)
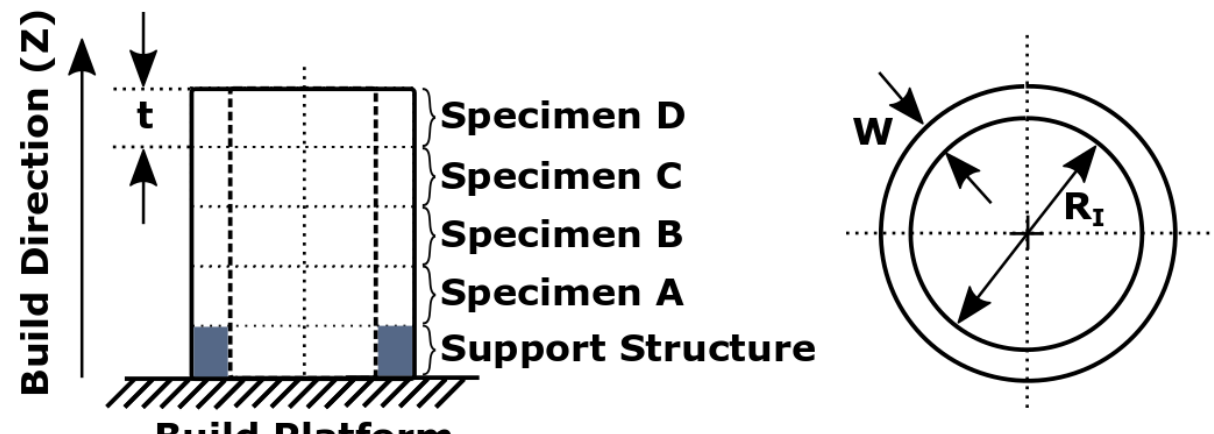

Build Platform

(b)

Figure 2: Figures illustrating the manufacture of small ring specimens and the labelling conventions used in the present work. A comparison of the final small ring specimen and tube in is shown in (a), with a schematic of the tube sectioning procedure shown in (b). Note that scaffold sections are discarded and tubes are sectioned by turning on a mandrel. General specimen dimensions, namely thickness $(t)$, width $(W)$, and internal radius $\left(R_{I}\right)$, are also shown in $(b)$.

\subsection{Experimental Methods and the Small Ring Test}

The small ring test set-up used in the present work can be summarised by figure 3 (showing both undeformed and deformed specimen states). In the test, a ring (approximately $10 \mathrm{~mm}$ in diameter) is loaded by two pins (allowing for self alignment and a minimal influence of friction on the specimen response) such that it may elongate. Tensile (imposed constant deflection rate) small ring tests are performed in the present work at room temperature by applying a constant displacement rate. $P$ is therefore recorded for changes in pin displacement $\left(\Delta-\Delta_{0}\right)$. 


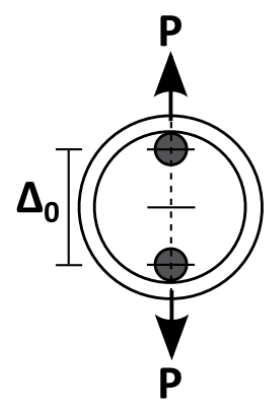

(a)

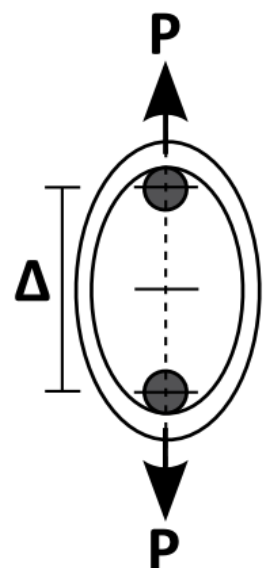

(b)

Figure 3: A schematic of the small ring testing method, showing (a) undeformed and (b) deformed specimens.

Small ring testing is completed here using a Tinius-Olsen H25KS test rig (see figure 4). Load is transferred from the machine end grips to the small ring sample through a modified Nimonic alloy test specimen which house Nimonic loading pins. Pin displacement is recorded through LVDTs connected to knife edge ridges on the modified Nimonic test specimen. The stiffness of the ring test specimen can be shown to be significantly less than that of the modified Nimonic "grip" sample for the majority of engineering materials, meaning that LVDT measurements taken from the grip can be assumed to be approximately equivalent to the pin displacements. A constant loading rate of $0.1 \mathrm{~mm} / \mathrm{s}$ is used in all tests.

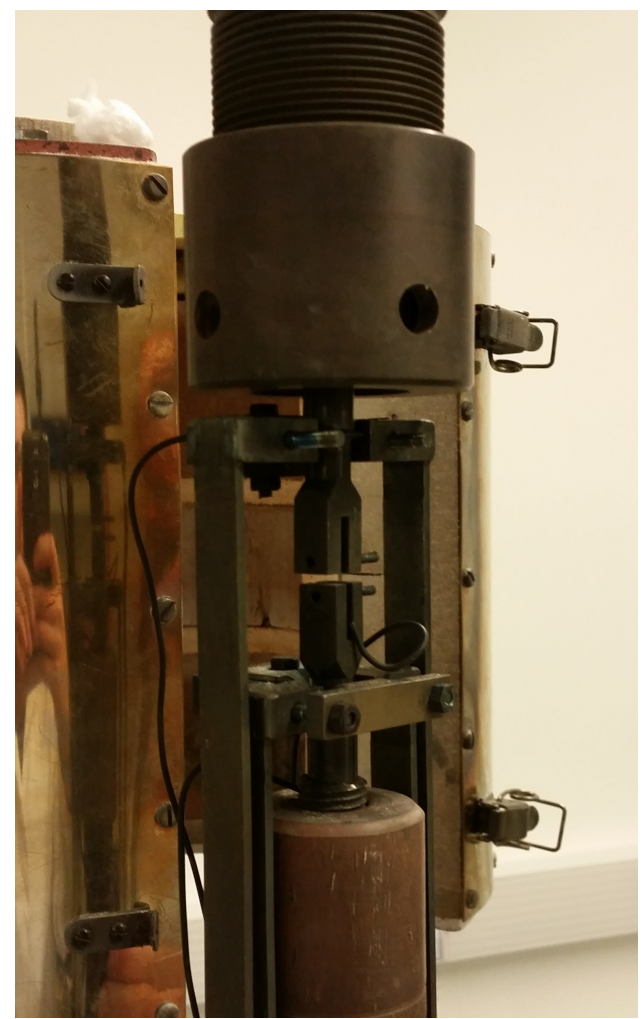

Figure 4: The Tinius-Olsen H25KS test rig used for small rig testing in the present work, showing a close up view of the modified small ring specimen grips. 


\section{Analytical Methodology}

\subsection{Overview of Parameter Determination Method}

An inverse method will be implemented in the present work in order to estimate local material properties from the results of small ring tests. The interpretation of small ring test data is, of course, more complicated than that of conventional uniaxial tests due to varying geometry of the sample and, consequently, the varying (equivalent) gauge length. In small ring testing non-linearity is observed in both the material and the geometry. When attempting to convert force/displacement type data to the equivalent uniaxial stress/strain data it is vital that both of these sources of non-linearity are accounted for if underlying material response is to be extracted correctly. It is often erroneously remarked that the onset of non-linearity in small ring force/displacement data is due to yielding, however it is easily shown (see Kazakeviciute et al. [31], for example) that plastic strain begins to accumulate long before this point. A reasonable approximation of non-linear stiffness may be achieved with a semi-analytical approach (such as the one developed in section 3.3), however accurate analysis of small ring test results is most readily achieved using finite element analysis. Both analysis strategies will be applied in the present work to interpret small ring data in a reliable and efficient manner.

The inverse method implemented here can be summarised by figure 5 . The following procedure is adhered to when one wishes to estimate the underlying constitutive behaviour from a set of small ring experimental results:

- Initial material parameter estimates are noted for the description of plastic (hardening) behaviour by considering conventional (full sized) test results. Note that these parameters do not need to be "good" estimates for the material in question. All that is required is that a consistent initial condition for hardening parameters (in the present work these are yield stress and ArmstrongFrederick parameters, see section 4.2) is established.

- An initial estimate for the Young's modulus is made directly from small ring experimental results by comparing linear elastic/non-linear geometry analytical model results to "initial" force/displacement responses. Analytical model results may be generated a priori for a given small ring geometry, allowing for rapid approximations of the elastic moduli to be made from the small ring test results.

- Initial estimates for material properties (relating to both elastic and plastic deformation mechanisms) are used as a starting point in a MATLAB based optimisation procedure, based around LSQNONLIN. In this procedure, a candidate set of material parameters are used to generate and run a finite element model of the small ring specimen/test. Numerical results are then compared to corresponding small ring experimental results by evaluating an objective function (section 4.2). Note that, due to potentially large differences in magnitude, material parameters values are normalised prior to optimisation.

- Candidate material parameter sets are incrementally updated based on maximum gradient optimisation algorithms implemented and the objective function is re-evaluated using the new, numerically estimated, force/displacement history.

- The optimisation process is terminated when one of several criterion is satisfied (these relate to the magnitude of changes in material parameters and objective functions between evaluations). The optimised parameter set is then taken as representative of the material test.

In the interest of clarity, the assumptions used in the present works modelling activities are summarised here:

- In initial models (used for basic interrogation of the experimental results), a linear isotropic/homogeneous material is assumed.

- In detailed finite element analysis (FEA) models (used iteratively in optimisation procedures), linearity is again assumed for elastic response representation and non-linearity (plasticity) is introduced in the material description through additively decomposed kinematic hardening terms. 
- Geometric non-linearity is incorporated in all models (analytic and numeric) by updating the specimen geometry incrementally during the solution.

- Castigliano's second theorem is used to develop analytical expressions which describe the stiffness of the small ring sample. Strain energy terms related to bending are formulated assuming an Euler-Bernoulli beam, meaning that through thickness shear is neglected (that is to say, the beam is regarded as "slender").

- Pin displacements considered in the present work are limited to $1.6 \mathrm{~mm}$. It is assumed (based on previous FEA investigations) that at this level of deformation affects such as anticlastic curvature can be neglected. The small ring deformation problem is therefore assumed to be represented by a plane stress approximation.

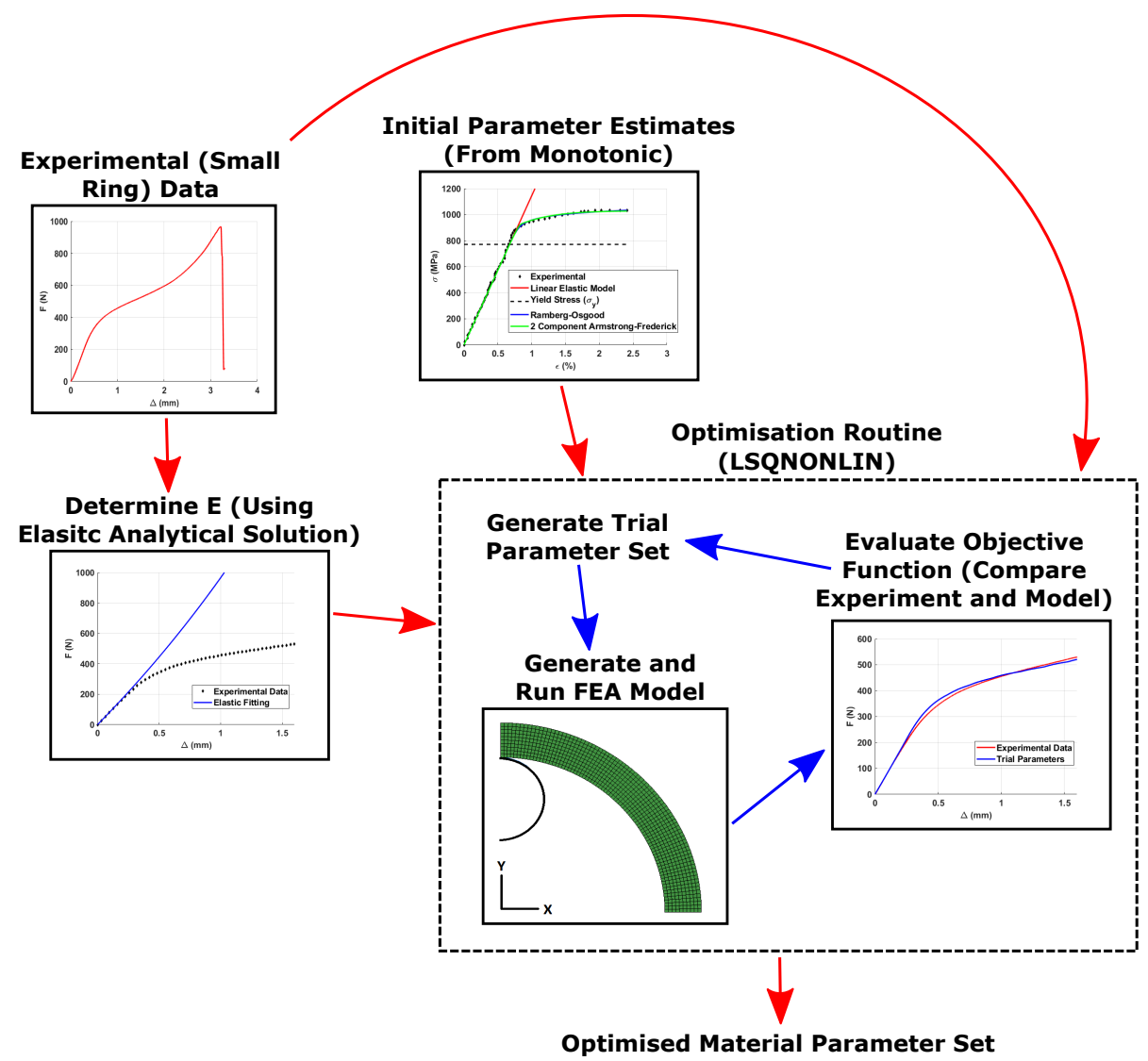

Figure 5: A flowchart summarising the parameter determination/ small ring interpretation method implemented in the present work. Note that single operations are indicated by red arrows and iterative procedures by blue arrows.

\subsection{Finite Element Modelling of the Small Ring Tensile Test}

Approximating the stiffness, and hence estimating the relationship between pin force and displacement, of a small ring sample can be most readily accomplished using the FEA method. By recalling the specimen dimensions used in the present work two alternative continuum FE formulations may be judged as appropriate, namely two dimensional plane stress and three dimensional ("brick") elements. It is clear that a full three dimensional representation of the small ring specimen is closer to reality as through thickness effects (such as anticlastic curvature, see Kazakeviciute et al. [31]) can be approximated. This additional fidelity comes at a computational cost which can quickly become significant in iterative procedures such as the inverse one implemented in the present work. Two dimensional plane stress 
approximations are usually cheaper than three dimensional equivalents (assuming similar levels of discretisation), thereby reducing the time taken per iteration. Previous work [31] has verified that, for the modest displacements considered in the present work, the difference between plane stress and full three dimensional elements representations is negligible. For this reason a quadratic shape function variant of the former is adopted here. In the commercial FEA system ABAQUS these quadratic plane stress full integration elements are denoted as CPS8. An approximate element side length of $0.1 \mathrm{~mm}$ is used in all cases (giving rise to approximately 800 elements over the quarter model) as, again, previous work has shown this is sufficient for convergence for the small ring geometry and for most engineering materials. Details of the mesh convergence study may be found in the authors previous work [31]. For reference, each elastic-plastic quarter ring FEA simulation (using the discretization described above) took approximately 10 minutes to complete when processed on a desktop machine utilising 2 cores in a $1.9 \mathrm{GHz}$ CPU with 8 GB of RAM.

Generalised python scripts have been developed which allow models to be generated, solved, and post processed (that is to say, force/displacement histories extracted) efficiently and with no manual intervention. Given that the stiffness of loading fixtures is significantly greater than that of the specimen, loading pins are modelled as analytically rigid surfaces in both modelling approaches. Reference points are associated with each rigid part (as is required in normal ABAQUS modelling methodologies). This allows all pin degrees of freedom to be constrained except for the loading direction (" $Y$ " translations in all cases). Reference points are also used to extract representative force/displacement histories from the model once solved. Note that the angular sizes of analytically rigid surfaces are limited in ABAQUS, meaning that pins cannot be modelled as $180^{\circ}$ semi-circular features. Excessive displacements will cause the ring to "wrap around" the pin, however the inclusion of a return curvature on the rigid pin means that contact conditions will not be compromised. No detrimental effects resulting from the inclusion of analytically rigid parts has been observed for the small displacement cases considered in the present work.

All planes of symmetry are exploited in the developed models by enforcing displacement boundary conditions (note that, in continuum elements, this also restricts related rotational degrees of freedom). In the case of the two dimensional plane stress assumption this reduces the model to a quarter of the ring geometry. Non-linear geometry is activated in all FEA models, regardless of material models implemented. An example of the complete FEA model, generated parametrically through ABAQUS' Python API, is presented in figure 6, with boundary conditions and the applied mesh highlighted in parts (a) and (b), respectively.

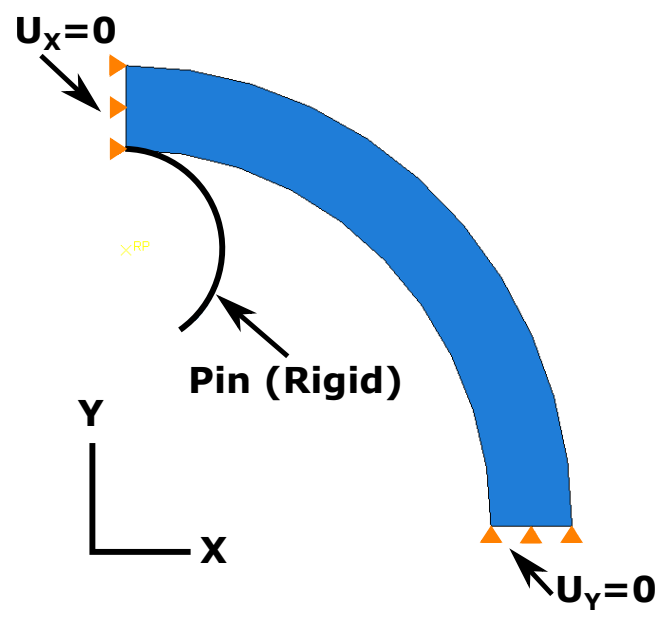

(a)

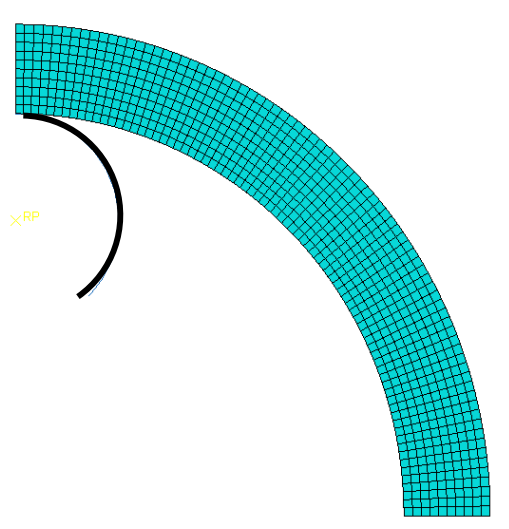

(b)

Figure 6: Plane stress (2D) FEA models of the small ring sample, showing (a) the application of boundary conditions and (b) the discretisation (CPS8 full integration elements) applied. 


\subsection{Analytical Model of the Small Ring}

In performing inverse analyses a set of initial material parameters must first be proposed. An analytical model is developed here which accounts for geometric non-linearity in a linear elastic solid by application of the well known principle of minimum total complementary strain energy and Castigliano's second theorem (these are excellently reviewed in the work of Reddy [36]). The analytical solution will be used in the inverse method to estimate Young's modulus, E, directly from experimental data without the requirement for iterative finite element analysis.

Non-linear geometry is accounted for in the energy based analytical model developed here by assuming the pin can be approximated by a point load (this is taken to be valid in the initial stages of ring deformation where elasticity is dominant) and the ring deforms to an ellipse under the application of load. By imposing symmetry only a quarter ring model is required, as shown in figure 7 (a), where $P$ is a vertical load (equivalent to the load measured in the small ring test), $Q$ is a horizontal dummy load that is applied so that horizontal displacements can be determined using the principle of virtual work, and $a$ and $b$ are the minor and major axis dimensions, respectively. Built in boundary conditions are applied to the horizontal plane of symmetry and a reaction moment, $M_{0}$, is applied on the vertical symmetry plane to enforce a zero gradient and replicate the constraint of the rest of the specimen. A free body diagram for an element of the analytical small ring model is shown in figure 7 (b). A segment of the small ring is defined by the angle $\theta$, at which point the effective radius of the ring, $R^{\prime}$, is defined by equation (1). The chord length at this point $(\gamma)$ can be expressed by equation (2), with the angle to the horizontal $(\zeta)$ and the angle to the tangent $(\phi)$ defined in equations (3) and (4), respectively.

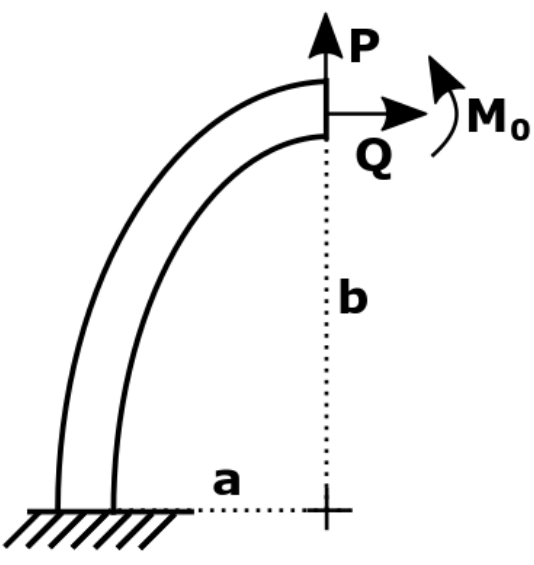

(a)

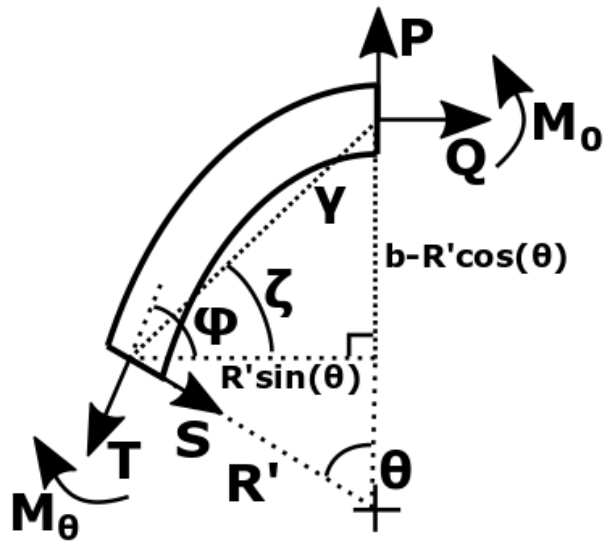

(b)

Figure 7: Definition of analytical model terms, showing (a) the elliptical ring geometry and (b), the free body diagram used to define internal forces and moments.

$$
\begin{array}{r}
R^{\prime}=a b \sqrt{\frac{\frac{a^{2}}{2}+\frac{b^{2}}{2}+\frac{\left(a^{2}-b^{2}\right) \cos (2 \theta)}{2}}{\gamma=\sqrt{b^{2}-2 b R^{\prime} \cos (\theta)+R^{\prime 2}}}} \\
\zeta=\tan ^{-1}\left(\csc (\theta)\left(\frac{b}{R^{\prime}}-\cos (\theta)\right)\right) \\
\phi=\tan ^{-1}\left(\frac{-b^{2}}{a^{2}} \tan (\theta)\right)
\end{array}
$$


The reaction moment at the coordinate $\theta, M_{\theta}$, can be expressed in terms of the loads $P, Q$, and $M_{0}$ by equation (5). This may then be used to calculate the complementary strain energy due to bending $\left(U_{B}^{*}\right)$, as shown in equation (6). By differentiating equation (6) with respect the load $M_{0}$ and equating the result to 0 (implying, by Castigliano's second theorem, that there is no rotation at the vertical line of symmetry) equation (7) may be developed, which expresses $M_{0}$ in terms of $P$ and $Q$. Several bending strain energy related integration terms, here noted by $A_{\alpha}$ (where $\alpha=1,2, \ldots, 6$ ), are used in equations (6) and (7). These are defined in equation (8). In the present work, integrals are approximated numerically using common trapezium rule approaches (here MATLAB's trapz function) so that increments in the ring geometry can be estimated (i.e. non-linearity in the ring geometry is approximated here by incrementally solving for force/displacement relationships and consequently updating ring dimensions $a$ and $b$ ).

$$
\begin{aligned}
& M_{\theta}=M_{0}+\gamma[P \cos (\zeta)-Q \sin (\zeta)] \\
& U_{B}^{*}=\int_{0}^{L} \frac{M_{\theta}^{2}}{2 E I} d x=\int_{0}^{\pi / 2} \frac{M_{\theta}^{2}}{2 E I} R^{\prime} d x \\
&=\frac{1}{2 E I}\left[M_{0}^{2} A_{1}+P^{2} A_{2}+Q^{2} A_{3}-P Q A_{4}+M_{0} P A_{5}-M_{0} Q A_{6}\right] \\
& M_{0}=\frac{Q A_{6}-P A_{5}}{2 A_{1}} \\
& A_{1}=\int_{0}^{\pi / 2} R^{\prime} d \theta \\
& A_{2}=\int_{0}^{\pi / 2} R^{\prime} \gamma^{2} \cos { }^{2}(\zeta) d \theta \\
& A_{3}=\int_{0}^{\pi / 2} R^{\prime} \gamma^{2} \sin { }^{2}(\zeta) d \theta \\
& A_{4}=\int_{0}^{\pi / 2} 2 R^{\prime} \gamma^{2} \sin (\zeta) \cos (\zeta) d \theta \\
& A_{5}=\int_{0}^{\pi / 2} 2 R^{\prime} \gamma \cos (\zeta) d \theta \\
& A_{6}=\int_{0}^{\pi / 2} 2 R^{\prime} \gamma \sin (\zeta) d \theta
\end{aligned}
$$

Tensile and shear forces ( $T$ and $S$, respectively) can be expressed in terms of the reaction forces $P$ and $Q$ by considering the free body diagram shown in figure $7(\mathrm{~b})$, leading to the development of equations (9) and (10). These can then be used to evaluate expressions for the complementary strain energy due to tension $\left(U_{T}^{*}\right)$ and the complementary strain energy due to shear $\left(U_{S}^{*}\right)$. Evaluations are shown in equations (11) and (12), respectively. Note that, in the case of $U_{S}^{*}$, the plane stress definition of shear modulus $G(G=E /(2(1+v)))$ is utilised. In a similar manner to bending strain energy contributions, integration terms for tensile and shear components, here noted by $B_{\alpha}$ (where $\alpha=1,2,3$ ), are given in equation (15).

$$
\begin{aligned}
& T=P \sin (\phi)+Q \cos (\phi) \\
& S=P \cos (\phi)-Q \sin (\phi)
\end{aligned}
$$




$$
\begin{gathered}
U_{T}^{*}=\int_{0}^{L} \frac{T^{2}}{2 E A} d x=\int_{0}^{\pi / 2} \frac{T^{2}}{2 E A} R^{\prime} d \theta \\
=\frac{1}{2 A E}\left[P^{2} B_{1}+Q^{2} B_{2}+P Q B_{3}\right] \\
U_{S}^{*}=\int_{0}^{L} \frac{S^{2}}{2 G A} d x=\int_{0}^{\pi / 2} \frac{S^{2}(1+v)}{E A} R^{\prime} d \theta \\
=\frac{(1+v)}{A E}\left[P^{2} B_{2}+Q^{2} B_{1}-P Q B_{3}\right] \\
B_{1}=\int_{0}^{\pi / 2} R^{\prime} \sin ^{2}(\phi) d \theta \\
B_{2}=\int_{0}^{\pi / 2} R^{\prime} \cos ^{2}(\phi) d \theta \\
B_{3}=\int_{0}^{\pi / 2} 2 R^{\prime} \sin (\phi) \cos (\phi) d \theta
\end{gathered}
$$

An expression for total strain energy may be found by summing $U_{M}^{*}, U_{T}^{*}$, and $U_{S}^{*}$. This may then be differentiated with respect to the loads $P$ and $Q$ to find the vertical $\left(u_{v}\right.$, see equation (16)) and horizontal $\left(u_{h}\right.$, see equation (17)) displacements, respectively. Note that, due to it being a dummy load, $Q$ is set to zero after differentiation. Non-linearity is considered by incrementally solving the analytical solution and updating the ring dimensions accordingly. That is to say, for the $n^{\text {th }}$ increment, a small increase in load $P$ is considered (assuming a ring of dimensions $a^{n}$ and $b^{n}$ ) and $u_{v}^{n}$ and $u_{h}^{n}$ are calculated. Ring dimensions are updated for the next increment, such that $a^{n+1}=a^{n}-u_{h}^{n}$ and $b^{n+1}=b^{n}+u_{v}^{n}$. Increments in $P$ are made until a pre-defined limit value of $u_{v}$ is achieved. The level of load incrementation has been varied in order to verify convergence (for the specimens considered here, a $10 \mathrm{~N}$ load increment was found to be more than sufficient).

$$
\begin{aligned}
& u_{v}=P\left(\frac{A_{2}}{E I}-\frac{A_{5}^{2}}{4 E I A_{1}}+\frac{B_{1}(3+2 v)}{A E}\right) \\
& u_{h}=P\left(\frac{A_{5} A_{6}}{4 E I A_{1}}-\frac{A_{4}}{2 E I}-\frac{B_{3}(1+2 v)}{2 A E}\right)
\end{aligned}
$$

The analytical model described above has been compared to the FEA model predictions developed in section 3.2, see figure 8 . The effect of the slenderness of the ring has been investigated by varying the internal radius to width ratio, $R_{I} / w$. Note that a ratio of $R_{I} / w=5$ is representative of Ti- $6 \mathrm{Al}-4 \mathrm{~V}$ small rings used in the present work. In order to assist in comparing results, pin forces $(P)$ in figure 8 have been normalised with respect to the maximum pin load $\left(P^{*}\right)$ observed for each $R_{I} / w$ ratio. Furthermore, peak vertical deflections are scaled proportionally based on the $R_{I} / w$ ratio, thereby ensuring a similar level of deflection between test cases. In all analyses a linear elastic material is assumed (note that non-linearity in the force/displacement history results from the geometry), with Ti-6Al-4V representative elastic material parameters (Young's modulus of 114GPa and Poisson's ratio of 0.3) assumed.

Important observations may be made from section 3.2. An increasing stiffness non-linear response can be seen in the force/displacement history predicted by the analytical model, indicating that at least some of the expected geometric non-linearity is represented by the adopted incremental analytical solution. A clear difference between analytical and FEA model force can also be observed which increases with $u_{v}$ and decreases with $R_{I} / w$ ratio. The analytical model developed above makes use of classical Euler-Bernoulli beam bending theory in order to estimate complementary strain energies due to bending (note that this is the largest contribution to total complementary strain energy in the small ring case). Well known "slender" beam assumptions are therefore implicitly made in the analytical solution, meaning that through thickness shear contributions are neglected. This is clearly not the case in the 
FEA models, where small ring "beams" of course have a finite thickness. Such an explanation indicates why increasing the $R_{I} / w$ ratio in figure 8 , resulting in a more slender beam, leads to a reduction in the difference between analytical and FEA results. Consider now a correction factor, $f_{c}$, which quantifies the difference between analytical and FEA model predictions. That is to say, $f_{c}$ is the factor by which forces, for a given displacement, determined by the analytical method must be multiplied in order to match the corresponding FEA prediction. Study of figure 8 indicates that $f_{c}$ will be a function of $u_{v}$. Figure 9 shows how $f_{c}$ varies with $u_{v}$ for several distinctly different Young's modulus values, assuming $R_{I} / w=5$ (recall that this is the $R_{I} / w$ ratio for the Ti-6Al-4V small ring specimens used in the present work). Almost no relationship between $E$ and $f_{c}$ can be observed in figure 9, further supporting the hypothesis that discrepancies between analytical and FEA model predictions are due to through thickness effects.

The introduction of $f_{c}$ offers a simple method to incorporate through thickness effects into the analytical model. Given the intended application of the analytical model, namely to determine approximate values for Young's modulus directly from experimental small ring data prior to detailed optimisation, this approach is considered preferable over more detailed modelling approaches (note that these are discussed later). A two component exponential function, shown in equation (18), is used here to relate $f_{c}$ to $u_{v}$. The correction function parameters $\alpha_{i}$ (where $i=1,2,3,4$ ) shown in table 2 have been determined using the data shown in figure 9 (which is applicable to the specimen geometry used in the present work, $R_{I} / w=5$ ). That is to say, the correction function given by equation (18) is fitted to the data shown in figure 9. In the present work, this is achieved using the well known MATLAB function FMINCON.

$$
f_{c}=\alpha_{1} \exp \left(\alpha_{2} u_{v}\right)+\alpha_{3} \exp \left(\alpha_{4} u_{v}\right)
$$

Table 2: Correction factor $\left(f_{c}\right)$ function (see equation (18)) parameters used in the present work, valid for $R_{I} / w=5$.

\begin{tabular}{cc}
\hline Parameter & Value \\
\hline$\alpha_{1}$ & 0.986 \\
\hline$\alpha_{2}$ & 0.103 \\
\hline$\alpha_{3}$ & $3.567 \times 10^{-6}$ \\
\hline$\alpha_{4}$ & 4.995 \\
\hline
\end{tabular}




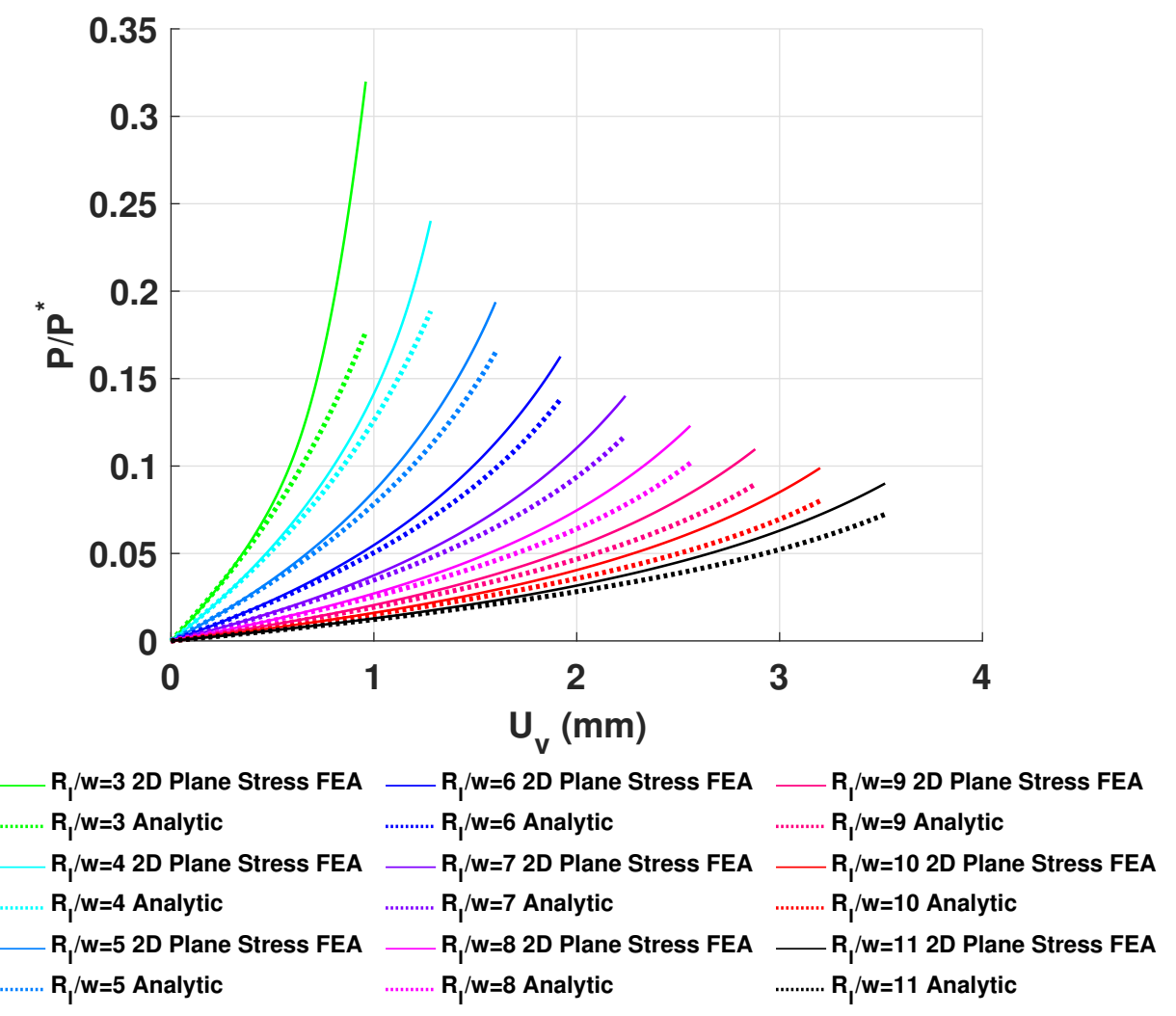

Figure 8: A comparison normalised force/displacement histories predicted by FEA (2D plane stress continuum elements) and analytical models for a range of ring geometries (specified by internal radius to width ratio, $R_{I} / w$ ). A linear elastic material is assumed in all cases, with a Young's modulus values of $114 \mathrm{GPa}$ and a Poisson's ratio of 0.3 . Note that $u_{v}$ is the calculated pin displacement, $P$ is the calculated pin reaction force, and $P^{*}$ in the maximum pin load observed for each $R_{I} / w$ ratio. Note also that a ratio of $R_{I} / w=5$ is applicable for the specimen geometries used in the present work.

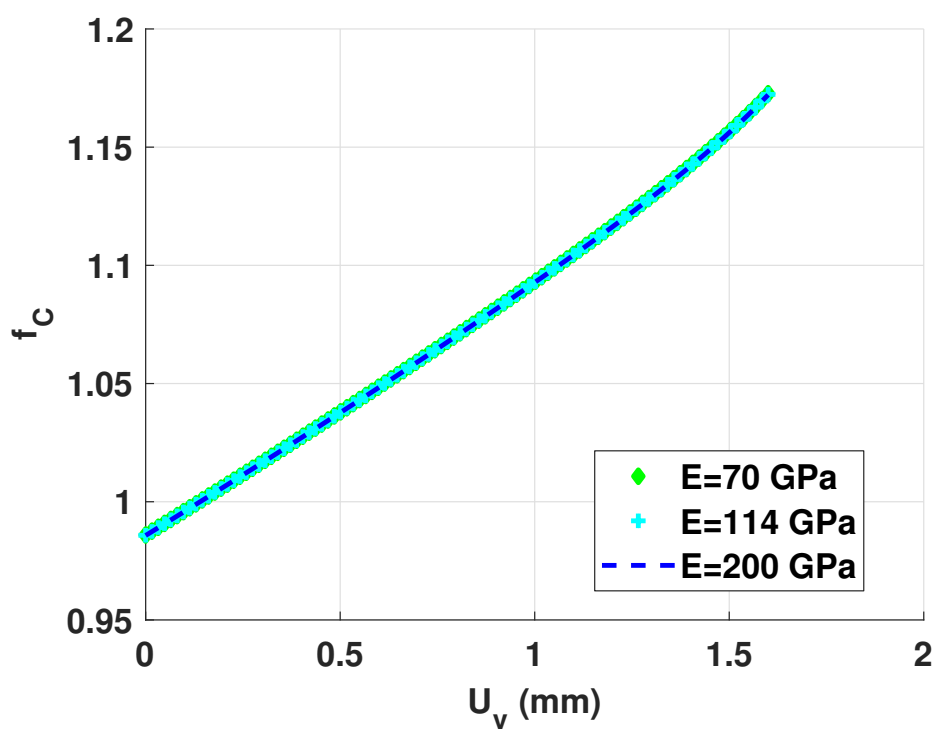

Figure 9: Correction factor $\left(f_{c}\right)$ values determined for $R_{I} / w=5$ for a range of Young's modulus values.

Trial force/displacement histories, determined a priori for a range of Young's modulus values using the analytical model and corrected to account for through thickness effects using $f_{c}$, must be 
compared to experimental small ring data in order to estimate Young's modulus. It is clear that the linear elastic material representation assumed in the analytical model will only be applicable for a limited range of experimental force/displacement histories; at some deformation the specimen response will be controlled by non-linearity in both the material (i.e. plasticity) and the geometry, rather than just the latter. The point at which material non-linearity begins to dominate specimen response is not immediately obvious from the experimental force/displacement history due to the effects of competing geometric non-linearity and interactions between material properties (particularly stiffness and yield stress). As such a robust, automated method to compare trial force/displacement histories to an experimental data set is required. The outcome of this procedure is the identification of a trial force/displacement history that best reflects the initial response observed in the given experimental data set (thereby indicating an approximate Young's modulus). This recommendation must of course be made without any knowledge of the material tested in the experiment.

Trial analytical solutions are compared to experimental data here using a simple sum of squares objective function, shown in equation (19), where $M$ is a number of data points (the number of data points in the experimental data set), $P_{i}^{\text {exp }}$ is the $i^{\text {th }}$ experimental load, and $P_{i}^{\text {num }}$ is the corresponding $\left(i^{\text {th }}\right)$ trial, analytical, solution. Note that, in practice, trial solution loads are interpolated at experimental displacements to allow for this comparison. A weighting, $W_{i}$ (see equation (20)), is applied to each sum of squares to bias differences at the start of the test (where elasticity should dominate specimen response). Equation (20) is logarithmically spaced using the biases $\left(\lambda_{u}\right.$ and $\left.\lambda_{l}\right)$ given in table 3 . After comparing all trial solutions the optimum solution is determined by searching for the minimum evaluation of equation (19).

Note that, in the present work, experimental data sets are limited to displacements less than $1.6 \mathrm{~mm}$ in order to ensure that only elastic-plastic behaviours are considered, rather than the initiation of cracks, their growth, and the eventual failure of the specimen.

$$
\begin{gathered}
f_{\text {Obj }}^{\text {Analytic }}=\sum_{i=1}^{M} W_{i} \sqrt{\left(P_{i}^{\text {exp }}-P_{i}^{\text {num }}\right)^{2}} \\
W_{i}=10\left(\lambda_{u}-\frac{\lambda_{l}-\lambda_{u}}{M-1}(i-1)\right)
\end{gathered}
$$

Table 3: Weighting function parameter values (see equation (20)) used to compare analytical small ring model results to experimental data and thus estimate local Young's modulus.

\begin{tabular}{cc}
\hline Parameter & Value \\
\hline$\lambda_{u}$ & 9 \\
\hline$\lambda_{l}$ & 0 \\
\hline
\end{tabular}

\section{Results and Data Interpretation}

\subsection{Small Ring Testing Results}

A total of 48 small ring tests have been conducted for the present work and are presented in figure 10 . Testing methodologies have been summarised in section 2.2. Specimen build locations, both within the build plane and vertically from it, are defined in figure 2 (a) and (c), respectively. Note that, in order to assist reader comprehension, plotting colours relating to specific specimens are maintained throughout the remainder of the paper. Individual build plane location results (showing small ring force/displacement relationships and interpreted constitutive behaviours) can be found in appendix A.1. 


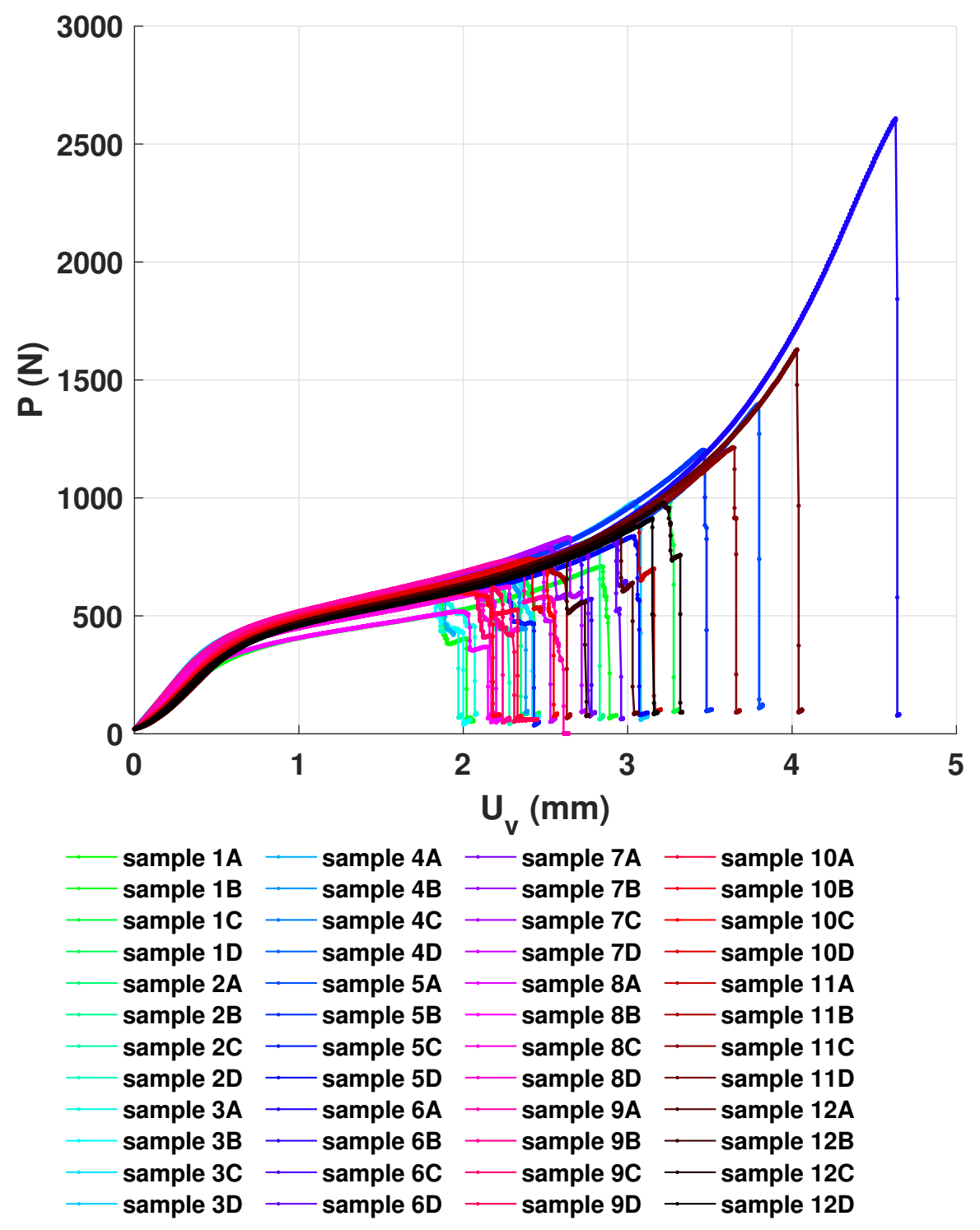

Figure 10: A summary of all Ti-6Al-4V experimental small ring results collected in the present work. Note that build plane locations and build height locations are defined in figure 2 (a) and (c), respectively.

\subsection{Initial Parameter Estimation for Hardening Description and Optimisation}

An elastic-plastic material model is required in order to describe material non-linearity in 2D plane stress FEA simulations the small ring tensile test. A simple elastic-plastic model is implemented here which assumes a von-Mises ( $J_{2}$ invariant) yield function and the normality hypothesis. A two component Armstrong-Frederick back stress law to describe kinematic hardening (which is particularly relevant for the description of monotonic behaviour). Total strain, $\boldsymbol{\epsilon}_{T}$, is decomposed in to elastic, $\boldsymbol{\epsilon}_{e}$, and plastic, $\boldsymbol{\epsilon}_{p}$, components (see equation (21)). Elasticity is assumed to follow Hooke's law as shown in equation (22), where $\sigma$ is the Cauchy stress tensor and $\mathcal{C}$ is the fourth order elastic stiffness tensor (isotropic elasticity is assumed using Young's modulus $E$ and Poisson's ratio $v$ ).

$$
\begin{gathered}
\boldsymbol{\epsilon}_{T}=\boldsymbol{\epsilon}_{e}+\boldsymbol{\epsilon}_{p} \\
\sigma=\mathcal{C}:\left(\boldsymbol{\epsilon}_{T}-\boldsymbol{\epsilon}_{p}\right)
\end{gathered}
$$

Plastic strain, $\epsilon_{p}$, accumulates according to the normality hypothesis while obeying the consistency 
condition, as shown in equation (23). Note that rate terms are denoted by a dot above relevant quantities. When the yield function, $f$, is satisfied (i.e. $f=0), \boldsymbol{\epsilon}_{p}$ accumulates in a direction normal to the yield surface (defined by the unit normal $\mathcal{N}$ ) by a scalar amount defined by $\lambda$ (the plastic multiplier). By substituting expressions for $f$, it may be shown that $\lambda$ is equivalent to the accumulated plastic strain $p$ (defined by equation (24)) and the deviatoric component of $\sigma, S$. A $J_{2}$ invariant based yield function is assumed, as shown in equation (25), where $\chi$ is the total back stress and $\sigma_{y}$ is the yield stress. A two component back stress decomposition is assumed, as shown in equation (26). Note that back stress decomposition allows for an improved representation of monotonic response, as discussed by Chaboche $[37,38,39,40]$. The $i^{\text {th }}$ back stress component is evolves by an Armstrong-Frederick rule [41], where $C_{i}$ is the $i^{\text {th }}$ component hardening modulus and $\gamma_{i}$ is a dynamic recovery exponent relating to the $i^{\text {th }}$ back stress component $\left(\chi_{i}\right)$.

$$
\begin{array}{r}
\dot{\boldsymbol{\epsilon}}_{p}=\dot{\lambda} \mathcal{N}=\dot{\lambda} \frac{\partial f}{\partial \sigma} \\
=\frac{3}{2} \dot{p} \frac{S-\chi}{J_{2}(\sigma-\chi)} \\
p=\left(\frac{2}{3} \epsilon_{p}: \epsilon_{p}\right)^{1 / 2} \\
f=J_{2}(\sigma-\chi)-\sigma_{y} \\
\chi=\sum_{i=1}^{2} \chi_{i} \\
\dot{\chi}_{i}=C_{i} \dot{\boldsymbol{\epsilon}}_{p}+\chi_{i} \gamma_{i} \dot{p}
\end{array}
$$

The material model described above can be readily implemented in ABAQUS using the in built combined hardening model. The procedure described in section 3.1 indicates that initial estimates of Ti-6Al-4V elastic-plastic material parameters $\left(E, \sigma_{y}, C_{1}, \gamma_{1}, C_{2}\right.$, and $\left.\gamma_{2}\right)$ are required prior to small ring optimisation. Monotonic data for large "dog bone“ samples printed in Ti-6Al-4V by the LPBF process is presented in the authors previous work $[42,43]$ and will be used to determine these initial conditions. Build volume location and orientation appeared to have little effect on the mechanical response in this previous study [42,43], however it should be noted that specimen dimensions were considerably large than those implemented here. Furthermore it is important to note that conventional samples were not built in a reduced build volume system, therefore some deviation between full sized and small ring results may be attributed to the significantly different processing history. Initial conditions derived from this "conventional" data will subsequently be modified in the optimisation procedure so that FEA elastic-plastic model results match a given set of experimental data. For the sake of brevity the material parameter fitting procedure is omitted here, however it should be noted that it is based on Cottrell's stress partitioning method [44], uses a linear regression approach to determine the onset of plasticity, and may be found in the author's previous work [45, 46, 47]. The well known Ramberg-Osgood equation (see equation (28), where $E_{R O}, K_{R O}$, and $n_{R O}$ are fitting parameters) is used as a smoothing function in this procedure to limit the effect of noise in the experimental data. The parameters shown in table 4 have been determined using the full sized specimen data and plastic related parameters $\left(\sigma_{y}, C_{1}, \gamma_{1}\right.$, $C_{2}$, and $\gamma_{2}$ ) are used in the small ring optimisation procedure (figure 5). Note that previous work has indicated, for most engineering materials, the small ring tensile response has a negligible dependency on Poisson's ratio $v$ [31]. A comparison of elastic-plastic and Ramberg-Osgood model predictions to the conventional Ti-6Al-4V monotonic material data is presented in figure 11. Yield stress $\left(\sigma_{y}\right)$ and and linear elastic response are also plotted in the same figure.

$$
\epsilon_{T}=\frac{\sigma}{E_{R O}}+K_{R O}\left(\frac{\sigma}{E_{R O}}\right)^{n_{R O}}
$$


Table 4: Elastic-plastic material properties determined using "full sized" experimental results for additive manufacture Ti64, used as initial conditions in the FEA coupled small ring optimisation procedure.

\begin{tabular}{cc}
\hline Parameter & Value \\
\hline Young's Modulus, $E$ & $114.45 \mathrm{GPa}$ \\
\hline Poisson's Ratio, $v$ & 0.30 \\
\hline Yield Strength, $\sigma_{Y}$ & $771.27 \mathrm{MPa}$ \\
\hline$C_{1}$ & $2.11 \times 10^{6} \mathrm{MPa}$ \\
\hline$\gamma_{1}$ & $1.42 \times 10^{4}$ \\
\hline$C_{2}$ & $2.74 \times 10^{4} \mathrm{MPa}$ \\
\hline$\gamma_{2}$ & 249.23 \\
\hline
\end{tabular}

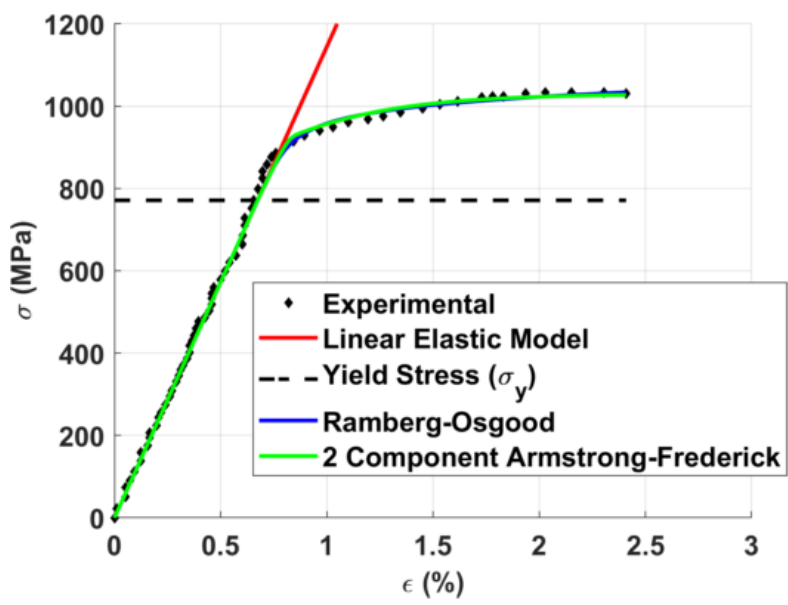

Figure 11: Experimental Ti64 monotonic data showing representative elastic-plastic behaviour for the LPBF Ti-6Al-4V material, taken from the work of Simonelli et al. [42, 43]. Predicted responses for elastic and elastic-plastic (a Ramberg-Osgood and a two component Armstrong-Frederick [41]) models are also presented, with the latter being used as initial conditions in small ring interpretation procedures. A reference value for yield stress $\left(\sigma_{y}\right)$ is also plotted.

The iterative optimisation procedure in figure 5 is implemented through the Levenberg-Marquardt function LSQNONLIN in MATLAB. This function acts to minimise the sum of squares objective function given in equation (29), $M$ is the number of experimental data points, $P_{i}^{\exp }$ is the $i^{t h}$ experimental load, and $P_{i}^{F E A}$ is the corresponding $i^{\text {th }}$ load predicted by the FEA model (using a trial set of material parameters). The optimisation procedure terminates when changes in the objective function between iterations fall below a tolerance $\left(1 \times 10^{-3}\right)$ or when the change in the material parameters is less than a tolerance $\left(1 \times 10^{-3}\right)$, both of which imply a local minimum. All material parameters are normalised prior to optimisation in order to ensure that the latter tolerance can be applied appropriately (i.e. modifications have a similar effect on material parameters that significantly different orders of magnitude). Back stress components are optimised such that they remain proportional. That is to say, only $C_{1}$ and $\gamma_{1}$ are modified in the optimisation procedure and $C_{2}$ and $\gamma_{2}$ determined using the $C_{1} / C_{2}$ and $\gamma_{1} / \gamma_{2}$ ratios expressed in table 4.

$$
f_{O b j}^{F E A}=\sum_{i=1}^{M} \sqrt{\left(P_{i}^{\exp }-P_{i}^{F E A}\right)^{2}}
$$

A comparison of small ring force/displacement curves and corresponding interpreted stress/strain behaviours (determined using the procedure outlined above) at each build plane location can be found in figures 14 to 25 in appendix A.1. 


\section{Discussion}

A good level of repeatability can be seen in both experimental data and local constitutive responses, as shown in figures 14 to 25 . The size of the printed tubes used to manufacture the small ring specimens limits how much vertical variation (i.e. perpendicular to the build plane) can be resolved in the small ring results presented here. No consistent trend is observed in experimental or constitutive results taken at specific build plane locations (i.e. over the vertical direction, with respect to the build direction figure 2). A summary of all constitutive responses, determined based on small ring tests conducted at the 48 build volume locations (i.e. the 12 tube locations on the build plane, each with 4 build direction locations), is presented in figure 12. Conventional test results, taken from section 4.2 (see table 4 ) in included for comparison, marked as "Reference".

It is important to note that, in figures 14 to 25 , experiential (force/displacement) data is linked to constitutive (stress/strain) data by the optimised material parameters only. That is to say, unlike in conventional uniaxial specimens, data interpretation does not assume a single stress or strain state for the specimen at any given load or displacement. Indeed, this is not possible given the biaxial nature of the small ring test. Gauge lengths and sections are therefore not calculated in the present work, although several other interpretation methods (see, for example Hyde et al. [30]) make use of this information. Equivalent gauge length is indeed a useful metric by which to compare small specimen methods as it indicates the sensitivity strain levels to changes in displacement. Note that the small ring boats a particularly high equivalent gauge length $(\geq 30 \mathrm{~mm})$, compared to other small specimen testing techniques. When implementing the inverse method assumptions must be made on the general constitutive behaviour of the material being tested. In the present case this is done when defining the material model (see section 4.2) and, for kinematic hardening, specifying the number and form (recall that Armstrong-Frederick is implemented here) of back stress terms. Conventional testing is used to determine this general form (and to determine initial estimates of elastic-plastic material parameters) for the AM Ti-6Al-4V material considered here. The small ring analysis method implemented here may, therefore, not be applicable for initial studies of a new material (for example). It is the case however that, if the general form of the constitutive response is known (for example, the number of required back stress terms is known), variations in properties may be easily explored using the small ring methodology.

An excellent level of repeatability is observed in small ring experimental data, with force levels in initial $(1.6 \mathrm{~mm})$ deformation of the ring in almost all cases being within $\pm 5 \%$ of mean (evaluated at a specific build location). An exception to this is build location 8 (see figure 21), wherein a much greater spread in data is observed ( $\pm 11 \%$ of mean). The increased scatter at build location 8 occurs in conjunction with a loss in ductility (all results in figure 21 (a) rupture at displacements less than $2 \mathrm{~mm}$ ). Indeed, figure 10 highlights the wide range of small ring specimen ductility. Note that all experimental data included in the figures in appendix A.1, however only the first $1.6 \mathrm{~mm}$ of deflection is used in the optimisation procedure. All deflection below $1.6 \mathrm{~mm}$ is assumed to be due to elasticity and material hardening, rather than the formation and propagation of cracks. By limiting optimisation data to 1.6 $\mathrm{mm}$ the complication of including failure mechanisms in the modelling approach is avoided. Future work could look to extend the method in this area, however extensive interpreted testing would be required in order to verify the onset of damage.

Post-test inspection of the small ring samples has revealed two main failure locations (in the ring geometry) and preliminary fractography has indicated that this is related to the level of porosity in the material. Samples that exhibit low ductility tend to fail near the loading pin and high levels of porosity are noted on fracture surfaces. FEA models indicate that large localised stress states occur near the loading pins due to the development of contact conditions in the early stages of the test. Large amounts of bending are also noted at the loading pins as the test progresses, leading to a tensile stress state at the outer ring surface. If significant levels of porosity exist in the sample it is suspected that pores will act as internal stress risers, thereby initiating cracks due to the tensile stress state and causing premature failure at the loading pin. In materials with lower levels of porosity the small ring has opportunity to develop in to an elongated ellipse, thereby leading to failure at between the pins (e.g. the planes of symmetry in figure 6) due to a combination of bending and tension. Note that, at this position in the ring, bending induced stresses are positive at the internal ring surface. Specimens that exhibit the failure mode are generally more ductile (see, for example, figure 18 for build location 5 ). The relationship 
between ductility and porosity leads to another potential application of the small ring test method for AM materials that warrants study in future work. The small ring method may be a convenient method by which to judge competing sets of print parameters when establishing a build. The specimens are simple and quick to print and test. Results could be gathered rapidly, with repartitions and across a build volume if required, and no microscopy/scanning would be necessary. In a similar way, small rings could easily be incorporated at the surfaces of printed components, leading local evaluations of properties for components and quality assurance.

The constitutive behaviours and material properties determined by the inverse approach have indicated a large degree of variation across the build volume. Considering a normal distribution of the basic elastic material parameters $E$ and $\sigma_{y}$, a mean value of $82.83 \mathrm{GPa}$ and a standard deviation of 4.57 $\mathrm{GPa}$ can be found for Young's modulus and a mean value of $655.58 \mathrm{MPa}$ and a standard deviation of $114.45 \mathrm{GPa}$ can be found for yield stress. The source of this variation may be investigated by determining median values for $E$ and $\sigma_{y}$ at each of the 12 build locations, as shown in figure 13. Consistent and higher $E$ and $\sigma_{y}$ values are noted along the left edge of the build volume (positions 1, 2, 3, 4, 5, 6, and 7), with large amounts of variation observed along the right edge. This is in agreement with the experimental results shown in figures 14 to 25 , wherein consistent and generally greater specimen ductilities are also noted at positions 1, 2, 3, 4, 5, 6, and 7. It is the suspicion of the authors that the variations observed in materials properties and specimen behaviour stem from the use of a RBV. Power is deposited from a hopper feeding from the top of the build volume (i.e. positions 3 and 8 are the first to be coated in a new powder bed) and there is a constant cooling air flow from left to right across the build plane. It is suspected that these factors cause asymmetry in the process history, thereby leading to the different mechanical responses. Future work will look to explore this point more closely by repeating the study with conventional build volume systems.

A marked difference is noted between material properties derived from conventional "dog bone" specimens (as in the author's previous work $[42,43]$ ) and materials properties derived from small ring results (as in the present). This warrants discussion, however it should be noted that there are many potential sources of this discrepancy, all of which should be investigated in future work. Firstly, it should be recognised that conventional specimen results, such as those presented in figure 11, were not manufactured using an RBV arrangement. The small rings used in the present work were, however, manufactured from tubes built in an RBV. Anecdotal evidence suggests that this will produce a more variable material over the build volume, and intuition indicates that processing history will be significantly different between RBV and conventional arrangements. Future work will repeat the small ring testing conducted here for conventional build volume tubes in order to provide insight here. Porosity and oxide formation have also been observed in fractured small ring samples and, while the levels of porosity are not sufficient to account for the lower Young's modulus observed (through a simple reduction in cross sectional area), it should be noted that point defects will modify the stress/strain states induced in the specimens. The presence of defects in a specimen will cause a load redistribution, thereby resulting in a stress state that potentially differs significantly from that observed in a homogeneous material. In samples with small gauge volumes (such as small rings) there is a reduced capacity for homogenisation, meaning that the effects of the localised defects are emphasised. Neglecting or including the effects of local defects will result in different reaction force/displacement relationship for some given constitutive behaviour, thereby influencing the outputs of inverse methods material parameter determination methods (such as those used in the present work). Work is underway to investigate the influence of local defects on small ring specimen results through assessment of the sensitivity of small ring material volume elements on overall specimen response and through pre and post test analysis of specimens. It is worth noting here the relevance of "thin" specimen results, as demonstrated in the recent work of Benzing et al. [48]. This work also considered AM Ti-6Al$4 \mathrm{~V}$ and a significant difference between thick "bulk" and thin "wafer" specimen results was noted, leading to different estimations of material properties. By considering thin specimens and limiting the gauge volume, it is possible to investigate which defects and heterogeneities are likely promote local deformation and premature failure. This is likely to be of great interest in many AM designs as bulk volumes are often replaced by infilled shells, leading to a requirement for thin section behaviour understanding. 


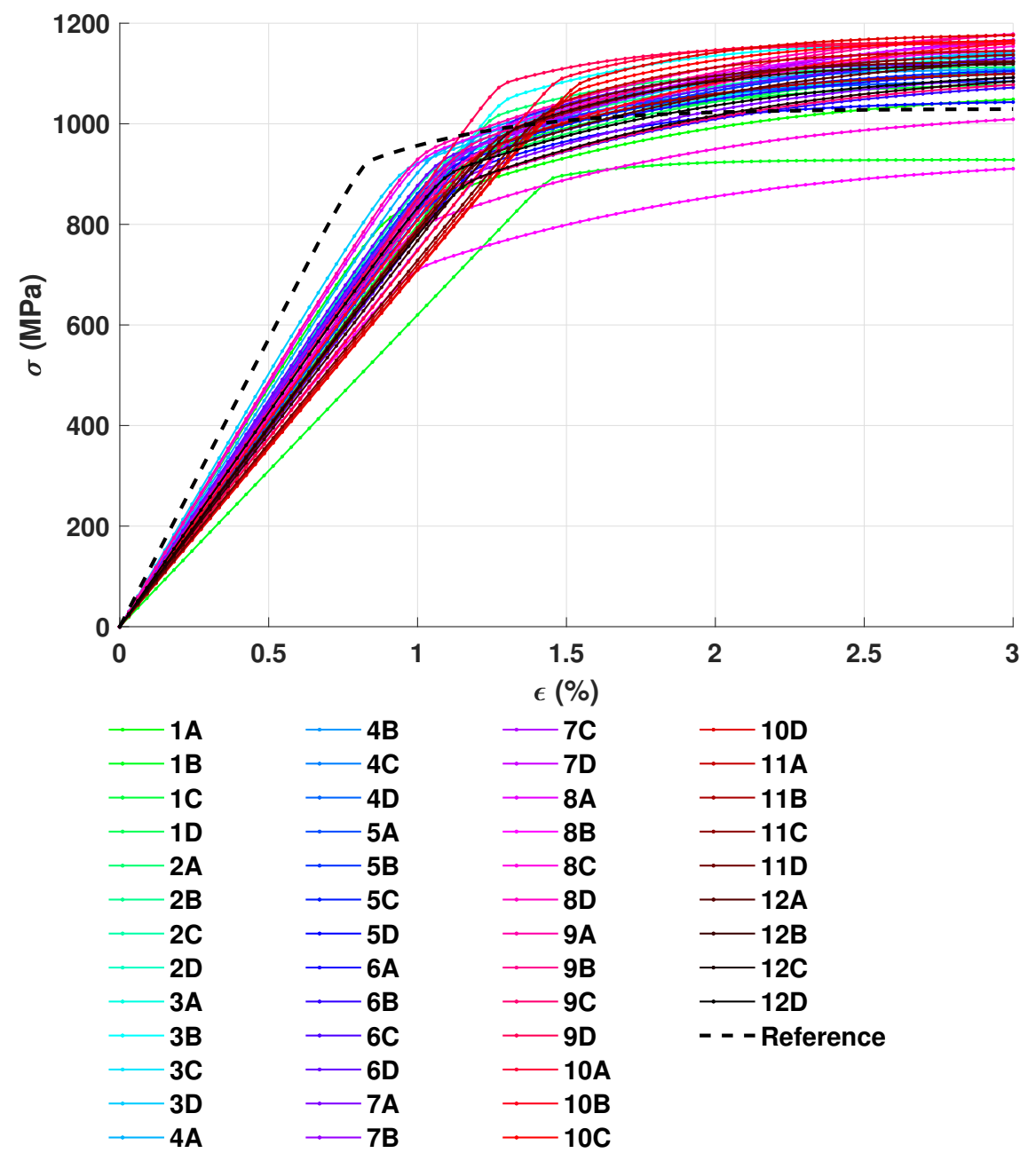

Figure 12: A summary constitutive responses (derived from optimised material property sets) for each print volume location. Note reference "full sized" results are also presented for comparison. These are determined in section 4.2 and summarised in table 4.

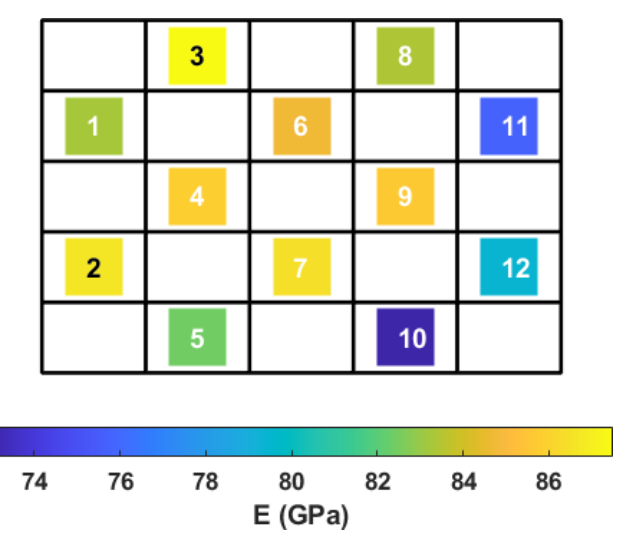

(a)

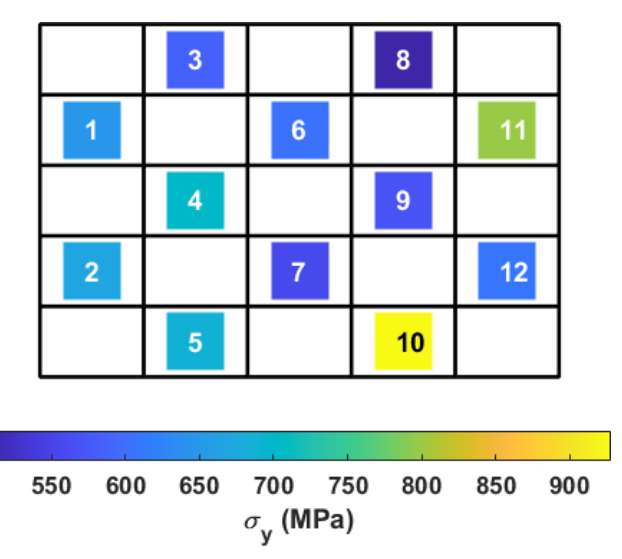

(b)

Figure 13: A comparison of median Young's modulus ( $E$, shown in (a)) and yield stress $\left(\sigma_{y}\right.$, shown in (b)) values at the twelve build volume locations. 


\section{Conclusions}

- Small ring specimens, manufactured via laser powder bed fusion, have been successfully tested and analysed to produce tensile data for a Ti-6Al-4V material. Small ring geometries boast several advantages over other small specimen types, including large equivalent gauge length dimensions (almost equivalent to most conventional specimen geometries) and self-aligning nature (under tension). An excellent level of repeatability is observed in small ring deformation behaviours at specific build plane locations, suggesting a robust testing technique which is dependent on local material properties.

- A strain energy based linear elastic analytical model has been developed for the small ring specimen which, along with an implementation method, can be used to derived approximate values for Young's modulus $(E)$ directly from experimental data. Discrepancies between the analytic solution and numerical (finite element) equivalents are attributed to the assumption of negligible through thickness shear in classical Euler-Bernoulli beam bending theory. A simple correction factor approach has been adopted here to account for the discrepancy as this strategy does not lead to any real additional computational effort and is easily implemented. Future work should look to extend the analytical model using the beam bending theory of Timeshenko (to correct through thickness effects in complementary strain energy terms [36]). The linear material model could also be refined to include nonlinearity (plasticity), as in the work of Szuladzinski [49].

- An optimisation procedure has been established which treats material parameter determination using small ring experimental data as an inverse problem. Initial conditions are derived from the analytical model. These are "fine tuned" by an optimisation procedure which generates trial finite element models (using test sets of material parameters) and compares numerical results to the experiential equivalent. The level of repeatability observed in experimental data if reflected in the derived constitutive responses, suggesting a robust data interpretation procedure.

- Variations in mechanical material properties has been observed in all 3 dimensions of the reduced build volume (RBV), however no consistent vertical (normal to the build plane) variations are observed between in build plane locations. Variation in basic material properties $E$ and $\sigma_{y}$ has been investigated over the build plane by taking median values for results at specific build plane locations (i.e. over all build height locations). Using this method, it was found that all $E$ values were within $\pm 11.91 \%$ and all $\sigma_{y}$ values were within $\pm 41.53 \%$.

- Further work required in order to progress the present study includes the validation of the method developed here for other LPBF materials and build conditions. The study will be repeated with a standard build volume as it is expected that the use of a RBV introduces significant scatter in the experimental data (preliminary fractography has indicated a large range in porosity over the build volume, for example). Other small specimen techniques, such as small punch, should also be investigated for additive material testing applications. The small ring geometry itself should also be the focus of study. Dimensions used here are those established in previous work and are largely controlled by "scoop" excavations taken from in service pressure bearing components. Additive manufacture of course relaxes this constraint, therefore this flexibility should be explored. In particular, non-circular rings should be investigated as increasing wall thickness at the loading pins will not detrimentally affect gauge length but may protect against premature failure of the specimen at the loading rings.

\section{Acknowledgements}

The work presented here has been made possible by funding provided through the Nottingham Research Fellowship programme.

\section{References}

[1] Schobeiri MT. Gas turbine design, components and system design integration. Springer; 2018. 
[2] Energy Roadmap 2050. European Commission; 2012.

[3] Gibson I, Rosen DW, Stucker B. Additive manufacturing technologies: rapid prototyping to direct digital manufacturing. Springer; 2010.

[4] Wang F. Mechanical property study on rapid additive layer manufacture Hastelloy X alloy by selective laser melting technology. International Journal of Advanced Manufacturing Technology. 2011;58(5).

[5] Wang X, Gong X, Chou K. Review on powder-bed laser additive manufacturing of Inconel 718 parts. Proceedings of the Institute of Mechanical Engineers Part B: Journal of Enginnering Manufacture. 2017;231(11).

[6] Amato KN, Gaytan MLEME S M, Shindo PW, Hernadez J, Collins S, Medina F. Microstructures and mechanical behavior of Inconel 718 fabricated by selective laser melting. Acta Materialia. 2012;60(5).

[7] Jia Q, Gu D. Selective laser melting additive manufacturing of Inconel 718 superalloy parts: densification, microstructure and properties. Journal of Alloys and Compounds. 2014;585.

[8] Rickenbacher L, Etter T, Hövel S, Wegener K. High temperature material properties of IN738LC processed by selective laser melting (SLM) technology. Rapid Prototyping Journal. 2013;19(4).

[9] Proöbstle M, Neumeier S, Hopfenmuüller J, Freund LP, Niendorf T, Schwarze D, et al. Superior creep strength of a Nickel-based superalloy produced by selective laser melting. Materials Science and Engineering A. 2016;674.

[10] Letenneur M, Kreitcberg A, Brailovski V. Optimization of laser powder bed fusion processing using a combination of melt pool modelling and design of experiment approaches: Density control. Journal of Manufacturing and Materials Proessing. 2017;3(21).

[11] Liu S, Shin YC. Additive manufacturing of Ti6Al4V alloy: A review. Materials and Design. 2019;164.

[12] Ettefagh AH, Zeng C, Guo S, Raush J. Corrosion behavior of additively manufactured Ti-6Al-4V parts and the effect of post annealing. Additive Manufacturing. 2019;28:252-258.

[13] de Formanoir C, Paggi U, Colebrants T, Thijs L, Li G, Vanmeensel K, et al. Increasing the productivity of laser powder bed fusion: Influence of the hull-bulk strategy on part quality, microstructure and mechanical performance of Ti-6Al-4V. Additive Manufacturing. 2020;33:101129.

[14] Villapún VM, Esat F, Bull S, Dover LG, González S. Tuning the mechanical and antimicrobial performance of a Cu-based metallic glass composite through cooling rate control and annealing. Materials. 2017;10(5).

[15] Parker J, James J. Creep behaviour of miniature disc specimens of low alloy steel. In: Pressure vessels and piping conference, Developments in a progressing technology. vol. 279; 1994. .

[16] Hyde CJ, Hyde TH, Sun W, Nardone S, De Bruycker E. Small ring testing of a creep resistant material. Materials Science and Engineering A. 2013;586.

[17] Hermosilla U, Hyde TH, Jones IA. Thermal analysis of electron-beam physical vapour deposited thermal barrier coated super-alloy tensile specimens. Proceedings of the Institution of Mechanial Engineers, Part L: Journal of Materials: Design and Applications. 2008;222.

[18] Hyde TH, Sun W, Becker AA, Williams JA. Creep behaviour and failure assessment of new and fully repaired P91 pipe welds at $923 \mathrm{~K}$. Proceedings of the Institution of Mechanical Engineers, Part L: Journal of Materials: Design and Applications. 2004;218.

[19] Xu Z, Hyde CJ, Tuck CJ, Clare AT. Creep behaviour of Inconel 718 processed by selective laser melting. Journal of Materials Processing Technology. 2018;256.

[20] Xu Z, Hyde CJ, Thompson A, Leach RK, Ravi GA, Maskery I, et al. Staged thermomechanical testing of Nickel superalloys produced by selective laser melting. Materials \& Design. 2017;133.

[21] Xu Z, Murray JW, Hyde CJ, Clare AT. Effect of post processing on the creep performance of laser powder bed fused Inconel. Additive Manufacturing. 2018;24.

[22] Xu Z, Dunleavey J, Antar M, Hood SL R Soo, Hyde CJ, Clare AT. The influence of shot peening on the fatigue response of Ti-6Al-4V surfaces subject to different machining and additive manufacturing processes. International Journal of Fatigue. 2018;111.

[23] Sanders M, Di Bella F, Liang H. Small-scale mechanical characterization of aluminum and titanium alloys. Journal of Testing and Evaluation. 2011;39(1).

[24] Husain A, Sehgal DK, Pandey RK. An inverse finite element procedure for the determination of constitutive tensile behaviour of materials using miniature specimen. Computational Materials Science. 2004;31(1-2).

[25] Holländer D, Kulawinski D, Thiele M, Damm C, Henkel S, Biermann H, et al. Investigation of isothermal and thermo-mechanical fatigue behavior of the nickel-base superalloy IN738LC using standardized and advanced test methods. Materials Science and Engineering A. 2016;67. 
[26] Han W, Yabucchi K, Kasada R, Kimura A, Wakai E, Tanigawa H, et al. Application of small specimen test technique to evaluate fracture toughness of reduced activation ferritic/martensitic steel. Fusion Engineering and Design. 2017;125:326-329.

[27] Shin CS, Lin SW. Evaluating fatigue crack propagation properties using miniature specimens. International Journal of Fatigue. 2012;43.

[28] Hyde TH, Hyde CJ, Sun W. Theoretical basis and practical aspects of small specimen creep testing. Journal of Strain Analysis for Engineering Design. 2012;48(2):112-125.

[29] Hyde TH, Hyde CJ, Sun W. A basis for selecting the most appropriate small specimen creep test type. Journal of Pressure Vessel Technology. 2012;136(2).

[30] Hyde TH, Sun W. A novel, high-sensitivity, small specimen creep test. Journal of Strain Analysis for Engineering Design. 2009;44:171-185.

[31] Kazakeviciute J, Rouse JP, De Focatiis DSA, Hyde CJ. The development of a novel technique for small ring specimen tensile testing. Theoretical and Applied Fracture Mechanics. 2019;99:131-139.

[32] Boyce B, Salzbrenner B, odelas J, Swiler L, Madison J, Jared B, et al. Extreme-value statistics reveal rare failure-critical defects in additive manufacturing. Advanced Engineering Materials. 2017;19(8):1-10.

[33] Salzbrenner B, Rodelas J, Madison J, Jared B, Swiler L, Shen Y, et al. High-throughput stochastic tensile performance of additively manufactured stainless steel. Journal of Materials Processing Technology. 2017;241:112.

[34] Lancaster RJ, Illsely HW, Davies GR, Jeffs SP, Baxter GJ. Modelling the small punch tensile behaviour of an aerospace alloy. Materials Science and Technology. 2016;33:1065-1073.

[35] Lancaster RJ, Jeffs SP, Illsley HW, Argyrakis C, Hurst RC, Baxter GJ. Development of a novel methodology to study fatigue properties using the small punch test. Materials Science and Engineering: A. 2019;748:21-29.

[36] Reddy JN. Energy principles and variational methods in applied mechanics. 3rd ed. Wiley; 2017.

[37] Chaboche JL. A review of some plasticity and viscoplasticity constitutive theories. International Journal of Plasticity. 2008;24:1642-1693.

[38] Chaboche JL. Time-independent constitutive theories for cyclic plasticity. International Journal of Plasticity. 1986;2:149-188.

[39] Chaboche JL, Rousselier G. On the plastic and viscoplastic constitutive-equations - part 1: Rules developed with internal variable concept. Journal of Pressure Vessel Technology - Transactions of the ASME. 1983;105:153158.

[40] Chaboche JL, Rousselier G. On the plastic and viscoplastic constitutive-equations - part 2: Application of internal variable concepts to the 316 stainless-steel. Journal of Pressure Vessel Technology - Transactions of the ASME. 1983;105:159-164.

[41] Frederick CO, Armstrong PJ. A mathematical representation of the multiaxial Bauschinger effect. Materials at High Temperatures. 2007;24(1):1-26.

[42] Simonelli M, Tse YY, Tuck C. Fracture mechanisms in high-cycle fatigue of selective laser melted Ti-6Al-4V. Key Engineering Materials. 2015;627:125-128.

[43] Simonelli M, Tse YY, Tuck C. Effect of the build orientation on the mechanical properties and fracture modes of SLM Ti-6Al-4V. Materials Science \& Engineering A. 2014;616:1-11.

[44] Cottrell AH. Dislocations and plastic flow in crystals. Oxford University, London. 1953.

[45] Rouse JP, Zacharzewski P, Hyde CJ, Jefferson-Loveday R, Morris A, Kyaw ST. A case study investigation into the effects of spatially dependent convection coefficients on the fatigue response of a power plant header component. International Journal of Fatigue. 2018;113.

[46] Kyaw ST, Rouse JP, Lu J, Sun W. Determination of material parameters for a unified viscoplasticity-damage model for a P91 power plant steel. International Journal of Mechanical Sciences. 2016;115-116:168-179.

[47] Rouse JP, Hyde CJ, Sun W, Hyde TH. Comparison of several optimisation strategies for the determination of material constants in the Chaboche visco-plasticity model. Journal of Strain Analysis for Engineering Design. 2013;48:347-363.

[48] Benzing JT, Liew LA, Hrabe FW N DelRio. Tracking defects and microstructural heterogeneities in meso-scale tensile specimens excised from additively manufactured parts. Experimental Mechanics. 2020;60:165-170.

[49] Szuladzinski G. Unit load method for deflections of planar, nonlinear structures. Journal of Mechanical Design. 1980;102:781-786. 


\section{A Appendices}

\section{A.1 Optimised Parameter Sets}

Results, both experimental (from the small ring tests, showing force/displacement histories) and numerical (constitutive responses determined by the analysis/optimisation procedure), are shown in figures 14 to 25 for build locations 1-12, respectively. All experimental data is presented (i.e. until failure), however only the initial $1.6 \mathrm{~mm}$ of deflection is used in optimisation procedures (as outlines in section 4.2). Constitutive responses are calculated numerically using the optimised material parameter sets, with peak strains limited to $3 \%$.

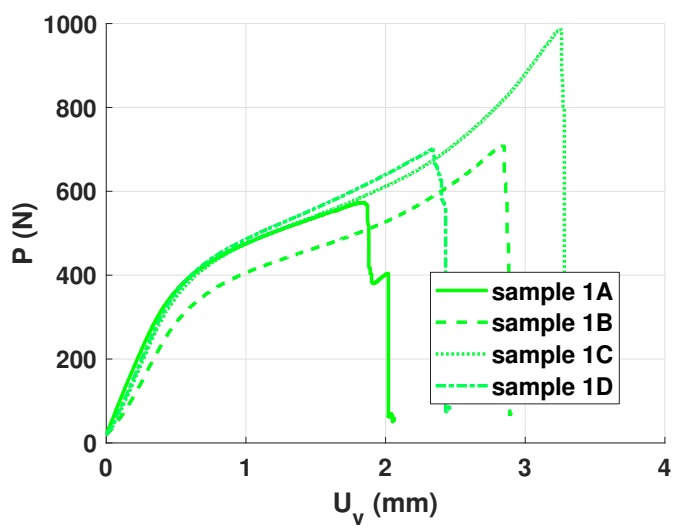

(a)

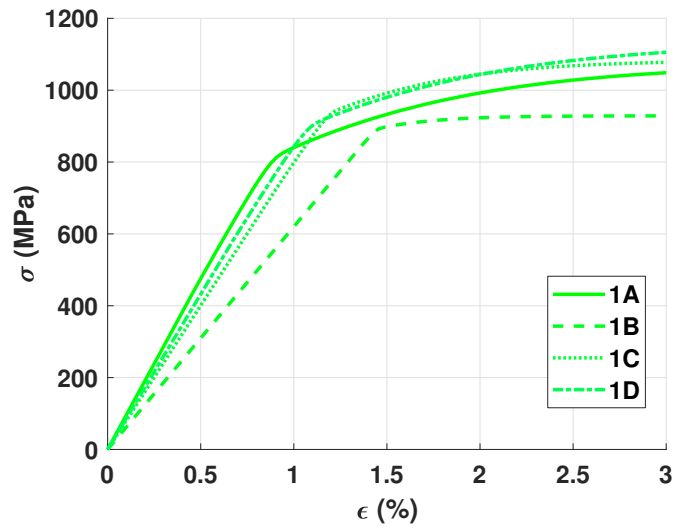

(b)

Figure 14: A comparison of experimental force/displacement data and interpreted stress/strain data for print bed position 1 (see figure 2 (a)), showing (a) the small ring data and (b) the constitutive behaviour interpreted by the method summarised in figure 5 .

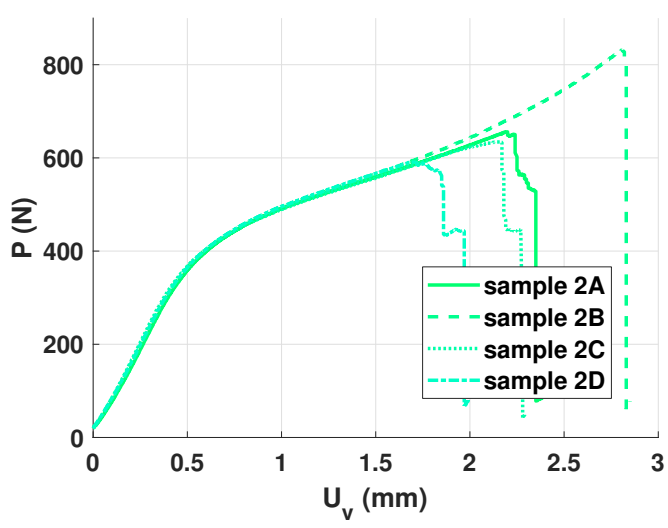

(a)

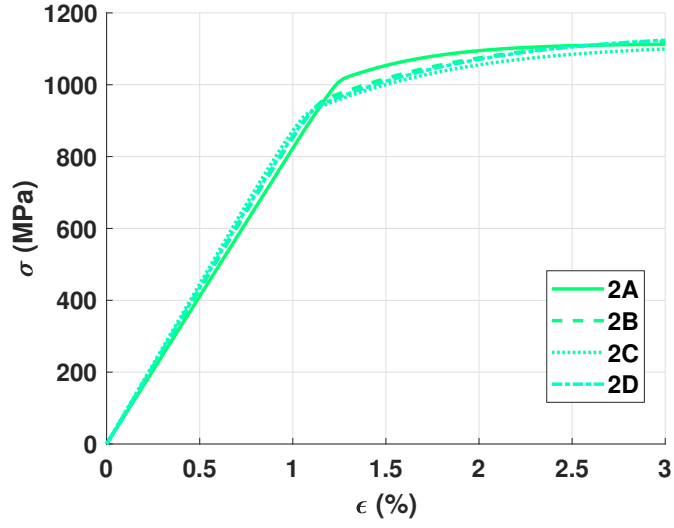

(b)

Figure 15: A comparison of experimental force/displacement data and interpreted stress/strain data for print bed position 2 (see figure 2 (a)), showing (a) the small ring data and (b) the constitutive behaviour interpreted by the method summarised in figure 5 . 


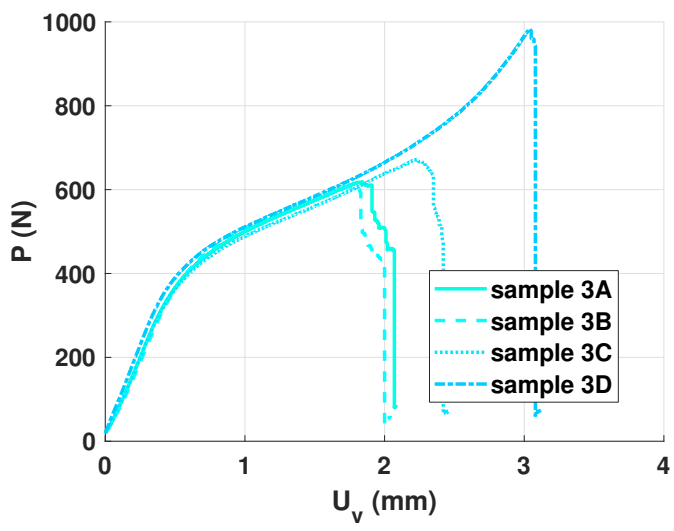

(a)

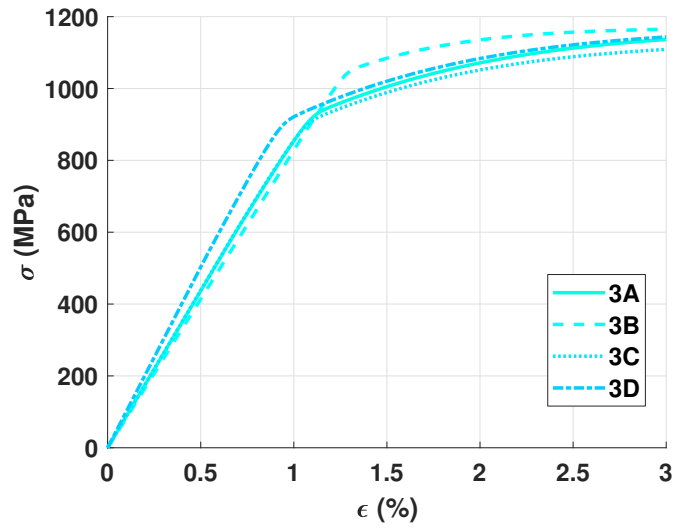

(b)

Figure 16: A comparison of experimental force/displacement data and interpreted stress/strain data for print bed position 3 (see figure 2 (a)), showing (a) the small ring data and (b) the constitutive behaviour interpreted by the method summarised in figure 5 .

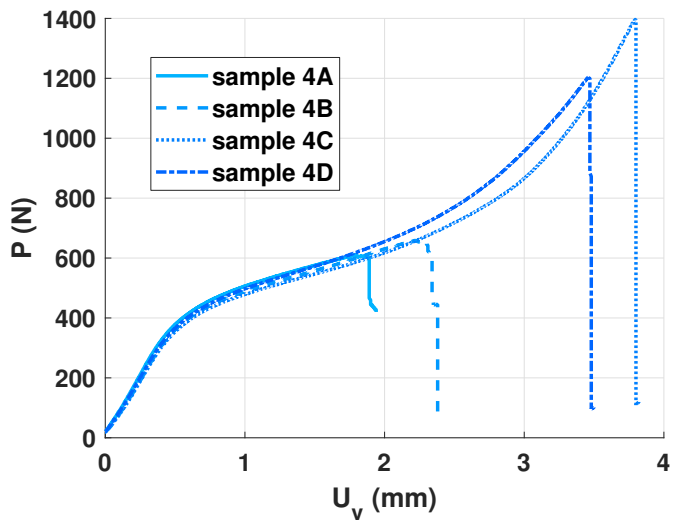

(a)

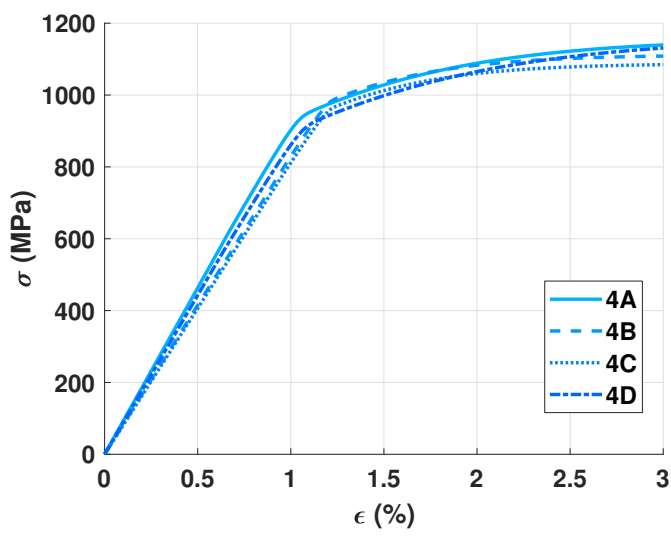

(b)

Figure 17: A comparison of experimental force/displacement data and interpreted stress/strain data for print bed position 4 (see figure 2 (a)), showing (a) the small ring data and (b) the constitutive behaviour interpreted by the method summarised in figure 5 . 


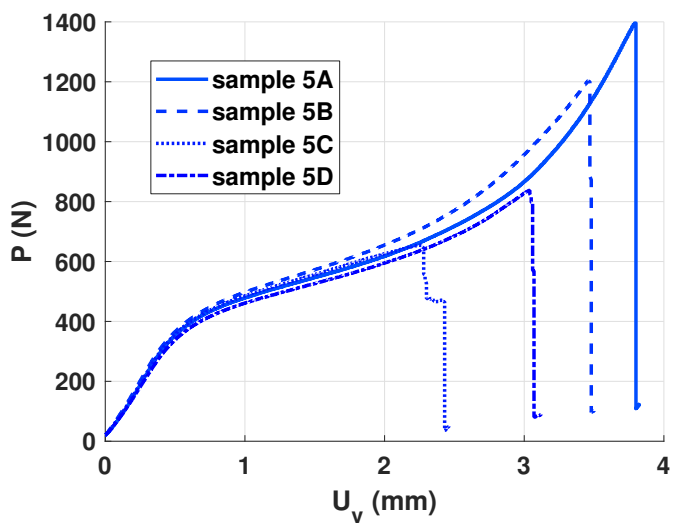

(a)

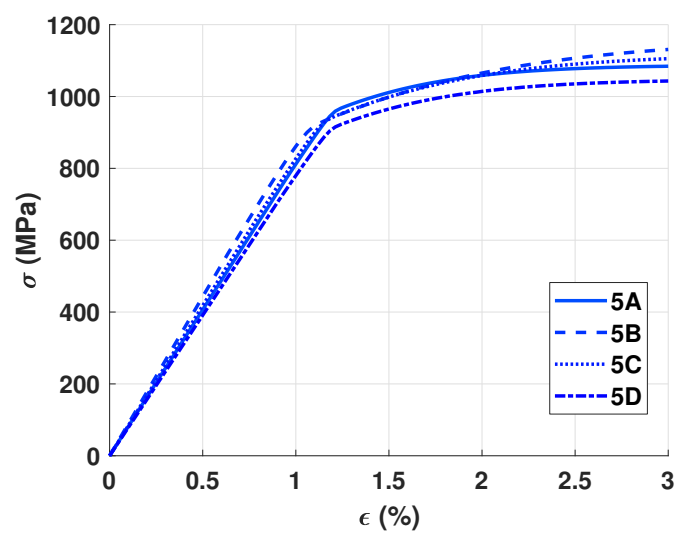

(b)

Figure 18: A comparison of experimental force/displacement data and interpreted stress/strain data for print bed position 5 (see figure 2 (a)), showing (a) the small ring data and (b) the constitutive behaviour interpreted by the method summarised in figure 5 .

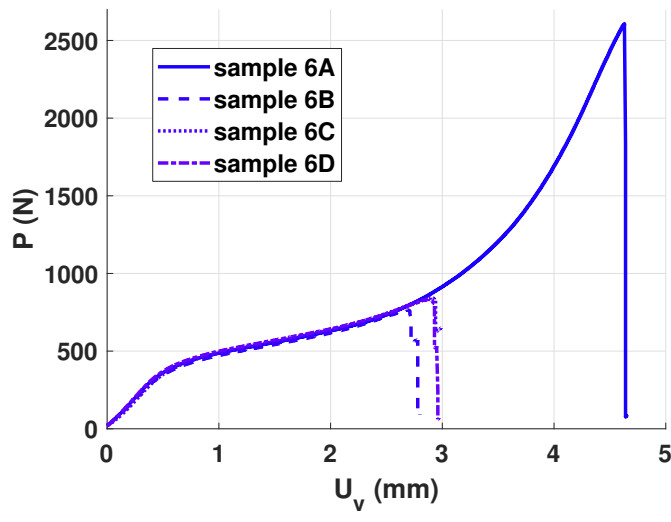

(a)

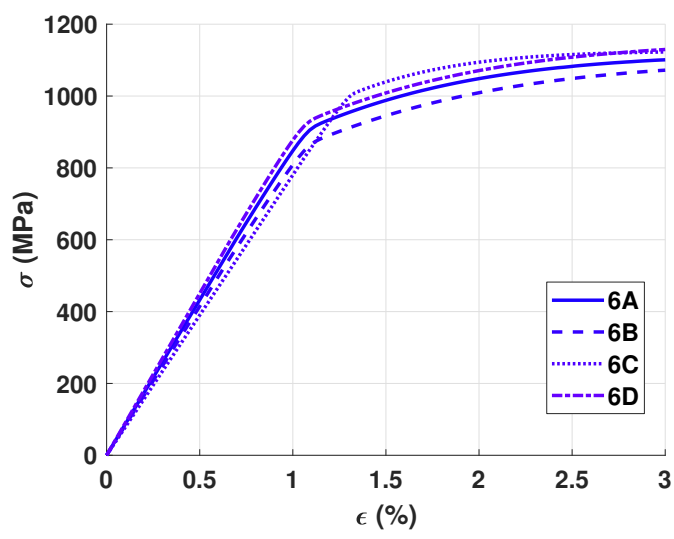

(b)

Figure 19: A comparison of experimental force/displacement data and interpreted stress/strain data for print bed position 6 (see figure 2 (a)), showing (a) the small ring data and (b) the constitutive behaviour interpreted by the method summarised in figure 5 . 


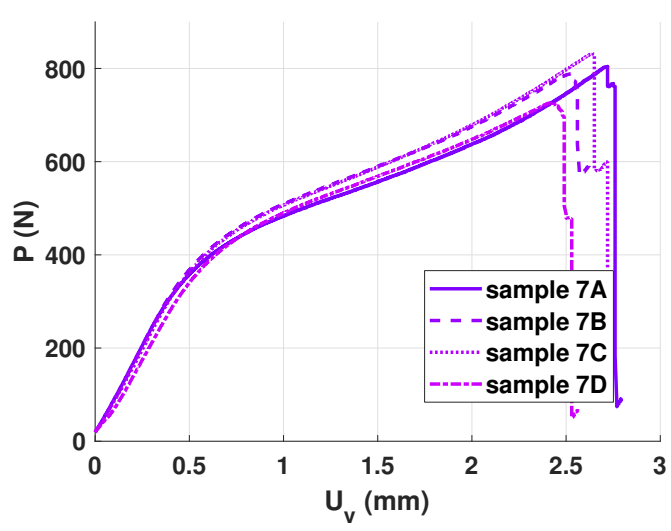

(a)

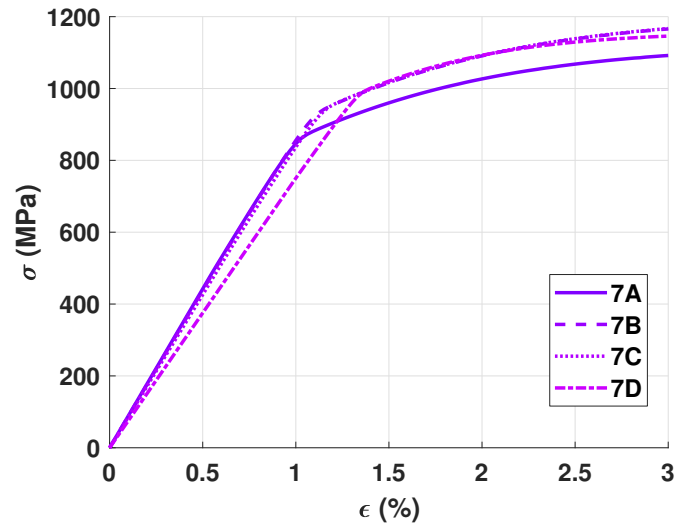

(b)

Figure 20: A comparison of experimental force/displacement data and interpreted stress/strain data for print bed position 7 (see figure 2 (a)), showing (a) the small ring data and (b) the constitutive behaviour interpreted by the method summarised in figure 5 .

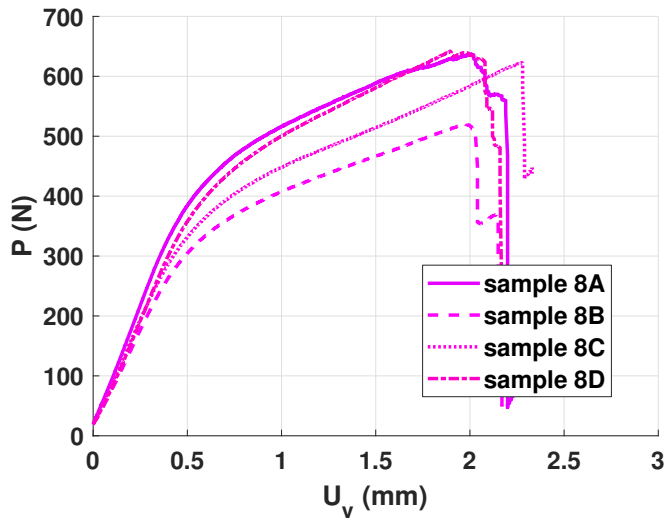

(a)

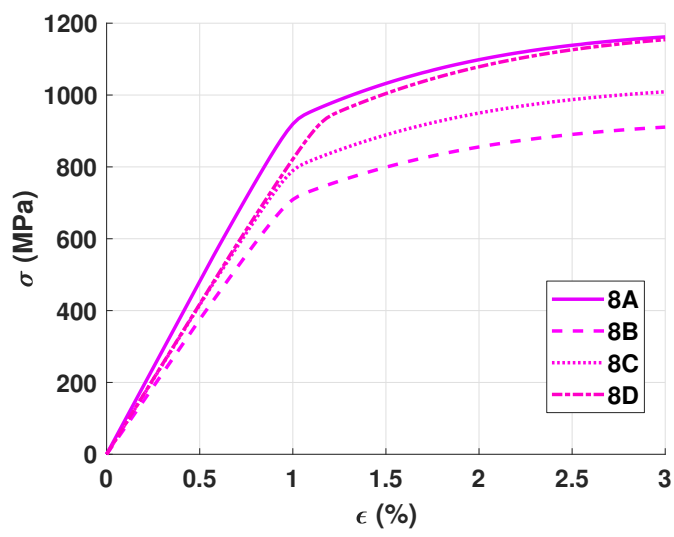

(b)

Figure 21: A comparison of experimental force/displacement data and interpreted stress/strain data for print bed position 8 (see figure 2 (a)), showing (a) the small ring data and (b) the constitutive behaviour interpreted by the method summarised in figure 5 . 


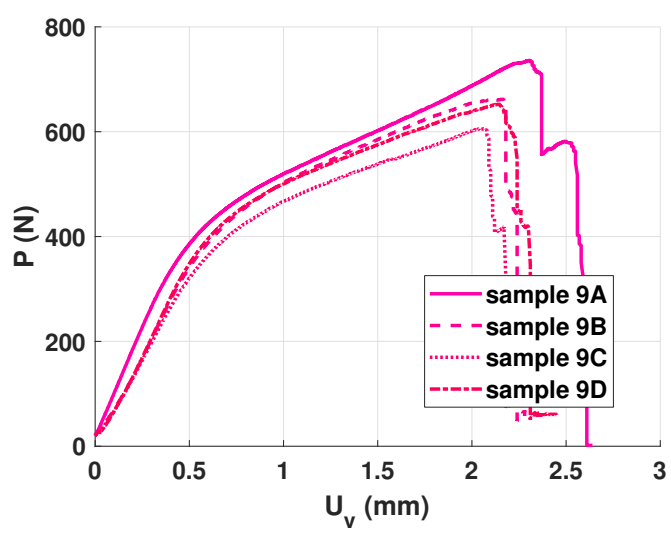

(a)

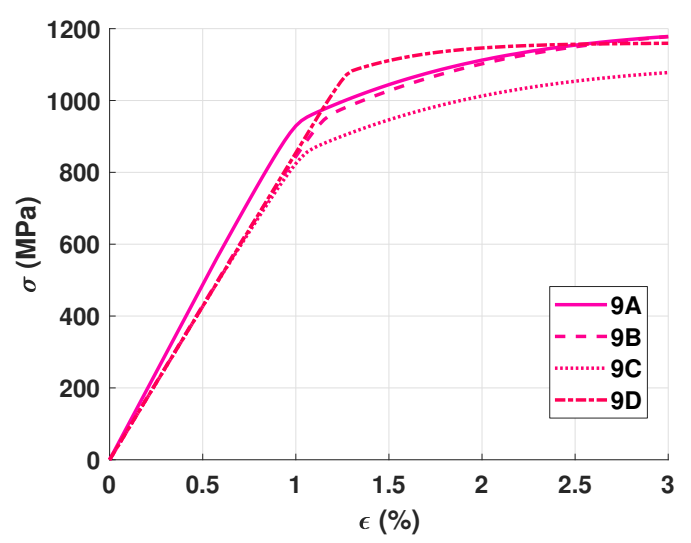

(b)

Figure 22: A comparison of experimental force/displacement data and interpreted stress/strain data for print bed position 9 (see figure 2 (a)), showing (a) the small ring data and (b) the constitutive behaviour interpreted by the method summarised in figure 5 .

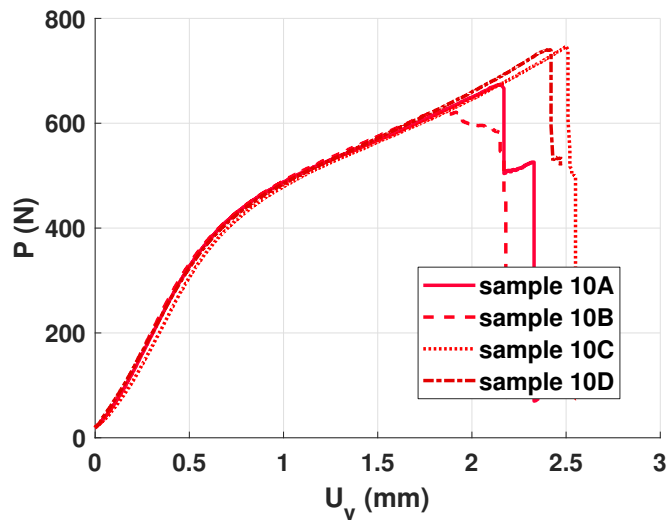

(a)

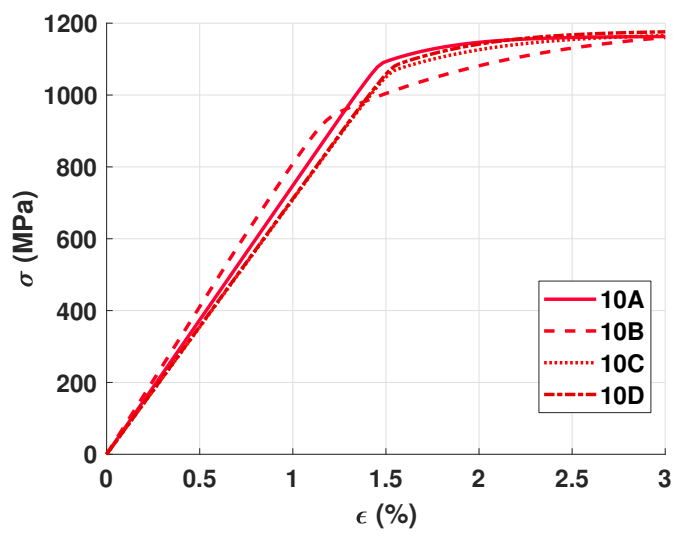

(b)

Figure 23: A comparison of experimental force/displacement data and interpreted stress/strain data for print bed position 10 (see figure 2 (a)), showing (a) the small ring data and (b) the constitutive behaviour interpreted by the method summarised in figure 5 . 


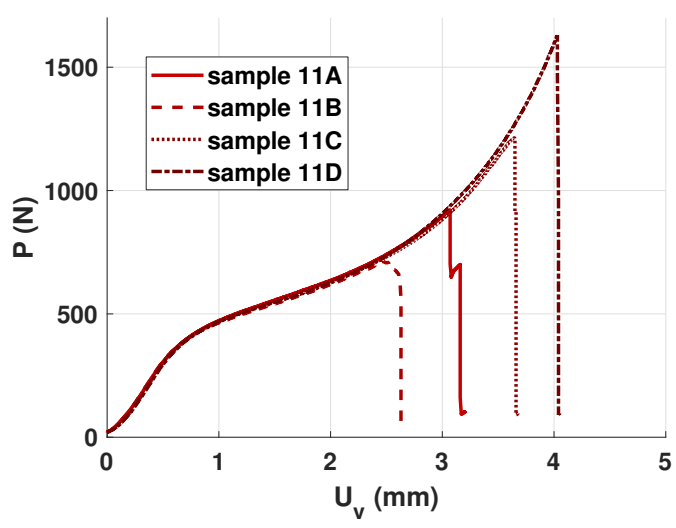

(a)

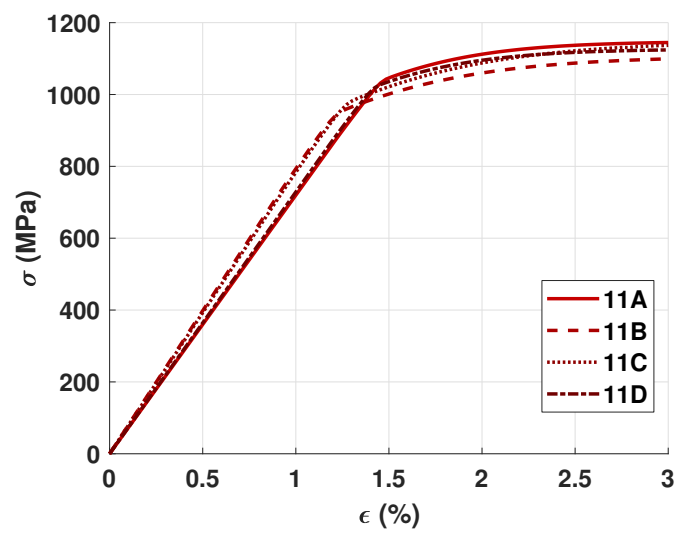

(b)

Figure 24: A comparison of experimental force/displacement data and interpreted stress/strain data for print bed position 11 (see figure 2 (a)), showing (a) the small ring data and (b) the constitutive behaviour interpreted by the method summarised in figure 5 .

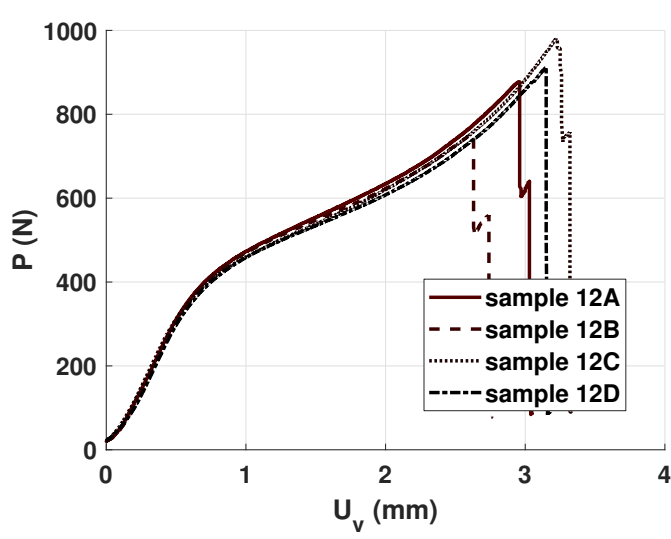

(a)

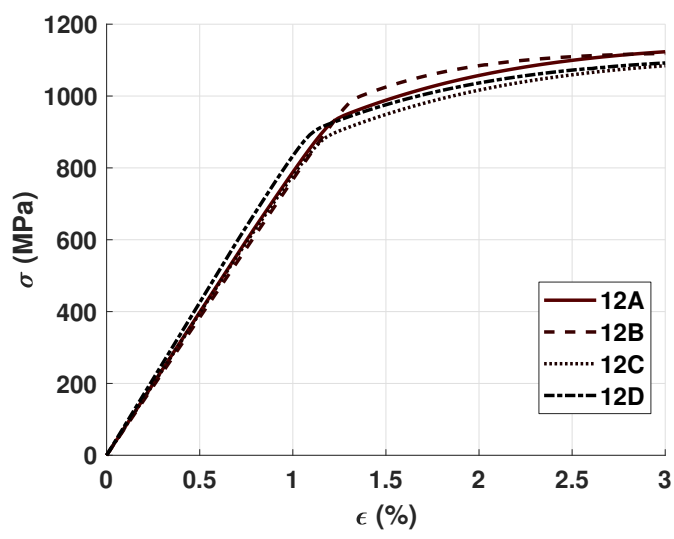

(b)

Figure 25: A comparison of experimental force/displacement data and interpreted stress/strain data for print bed position 12 (see figure 2 (a)), showing (a) the small ring data and (b) the constitutive behaviour interpreted by the method summarised in figure 5 . 